\title{
Desigualdades Ponderadas para Operadores Maximais
}

\author{
Antonio Luis Mometti
}

Orientador: Prof. Dr. Sérgio Luís Zani

Dissertação apresentada ao Instituto de Ciências Matemáticas de São Carlos, da Universidade de São Paulo, como parte dos requisitos para a obtenção do título de "Mestre em Ciências-Área: Matemática".

São Carlos, fevereiro de 1997. 
A minha Familia

e à minha Moiva. 


\section{Agradecimentos}

A Deus, pelo dom da vida.

Ao Prof. Dr. Sérgio Luís gani, pela atensão, dedicasão, amizade, incentivo e apoio durante o periódo de orientagão.

A minha familia, em especial aos meus pais Antonio e Rosa, e aos meus irmãos: Aucimar, Altair e Alaudenice, que sempre me apoiaram e almejaram exxito neste trabalho.

A minha nowa Ana Nery, pelo amor, carinho, compreensão e incentivo durante a elaboracão deste trabalho.

Ao meu grande amigo Marcio, pelo companheirismo e cooperacãa.

Aos professores do Instituto de Ciências Matemáticas de São Carlos, em especial a Ozíride Manzoli Neto, Maria Aparecida Soares Muas, Lres Dias, Valdir Antonio Menegatto, Luiz Augusto da Costa Ladeira e Alexandre Nolasco de Carwatho, pelo muito que aprendi com seus ensinamentos.

Aos professores do Instituto de Deociências e Ciências Exatas da UnESP - Dio Claro, pela formação que me proporcionaram.

Alos amigos de graduacão e pós-graduacão: Ana Claudia, Simone, Regina, Melson, Marta, Regilene e Katia, que foram companheiros de estudo e serão amigos para sempre.

Aos amigos mais recentes: André, Claudemir, Carlos, Anderson, Alexandra, Sarita, Luis Carlos, Adriana, Silvana, Luciane e Lauro, pelos momentos agradáueis que compartithamos.

A todos os funcionários do ICMSC, pela atensão e ficiência.

Ao CNPg, pelo suporte financeiro.

Enfim, agradeso a todas as pessoas que direta on indiretamente contribuiram para a realização deste trabahtho. 


\section{Abstract}

Maximal operators of Hardy-Littlewood and fractional types control the behavior of many other operators in Harmonic Analysis on weighted $L^{p}-$ spaces. One of these operators is the fractional integral.

The purpose of this thesis is to present a study on norm inequalities for maximal operators defined over starlike sets. 


\section{Resumo}

Operadores maximais dos tipos Hardy-Littlewood e fracionário controlam o comportamento de vários outros operadores da Análise Harmônica em espaços $L^{p}$ com pesos. Um desses operadores é a integral fracionária.

O objetivo desta dissertação é apresentar um estudo sobre desigualdades ponderadas para operadores maximais definidos sobre conjuntos estrelados. 


\section{Índice}

0 Introdução 1

1 Preliminares 6

2 As Classes de Pesos $A_{p}$ e o teorema de Muckenhoupt $\quad 10$

2.1 Função Maximal Homogênea de Hardy-Littlewood. . . . . . 10

2.2 As classes de pesos $A_{p}$ e o teorema de Muckenhoupt. . . . . . 14

2.3 Desigualdades ponderadas para o operador maximal centrado $M_{Q_{1}, \mu} \ldots \ldots \ldots \ldots \ldots \ldots \ldots$

3 Desigualdades ponderadas para operadores maximais sobre conjuntos estrelados

3.1 Desigualdades dos tipos forte e fraco para o operador maximal centrado $M_{S, \mu}$ e desigualdade do tipo fraco para o operador maximal não centrado $M_{S, Q_{1}, \mu}$, caso $S=S_{\rho} \ldots \ldots \ldots 29$

3.2 Desigualdades dos tipos forte e fraco para o operador maximal centrado $M_{S, \mu}$, caso $S$ estrelado em relação à origem. . . . . . 44

3.3 Desigualdade do tipo forte para o operador maximal não centrado $M_{S, E, \mu} \ldots \ldots \ldots \ldots \ldots \ldots$

3.4 Desigualdade do tipo fraco para o operador maximal não centrado $M_{S, E, \mu}$, caso $S$ estrelado em relação à origem. . . . . . 58 


\section{Capítulo 0}

\section{Introdução}

Seja $f$ uma função localmente integrável em $\mathbb{R}^{n}$. Definamos

$$
M f(x)=\sup _{Q} \frac{1}{|Q|} \int_{Q}|f(y)| d y
$$

onde o supremo é tomado sobre todo cubo $Q$ contendo $x$. Mf denota o operador maximal de Hardy-Littlewood. De acordo com um resultado de Muckenhoupt [M], Mf é um operador limitado sobre o espaço $L_{w}^{p}, 1<p<$ $\infty$, se e somente se o peso $w$ satisfaz a simples condição geométrica

$$
\left(\frac{1}{|Q|} \int_{Q} w d x\right)\left(\frac{1}{|Q|} \int_{Q} w^{-1 /(p-1)} d x\right)^{p-1} \leq c
$$

para todo cubo $Q$, denominada condição $A_{p}$.

$O$ estudo de tais propriedades para operadores maximais do tipo (0.1) tem sido de grande importância para o estudo da continuidade em espaços com peso dos operadores da Análise Harmônica Clássica. Um desses operadores é a integral fracionária definida por

$$
I_{\Omega, \mu} f(x)=f * K_{\Omega, \mu}(x),
$$

onde

$$
K_{\Omega, \mu}(x)=\frac{\Omega(x)}{|x|^{n-\mu}}
$$

para $0<\mu<n$ e para $\Omega$ uma função não negativa homogênea de grau zero. $\mathrm{O}$ kernel $K_{\Omega, \mu}$ tem o seguinte conjunto estrelado associado: seja $\rho(\theta)=$ $\Omega(\theta)^{1 /(n-\mu)}$ e seja

$$
S=S_{\rho}=\left\{r \theta ; \theta \in S^{n-1}, 0 \leq r<\rho(\theta)\right\}=\left\{x ; K_{\Omega, \mu}(x)>1\right\} .
$$


É claro que $S$ é um conjunto estrelado com relação à origem e que $|S|<\infty$ se e somente se $\rho \in L^{n}\left(S^{n-1}\right)$. Para $\Omega \equiv 1$ em $(0.2)$, temos

$$
I_{\mu} f(x)=f *|x|^{\mu-n}=\int_{\mathbb{R}^{n}} \frac{f(x-y)}{|y|^{n-\mu}} d y
$$

$I_{\mu}$ é denominada a integral fracionária de índice $\mu$.

Em $[\mathrm{MW}]$ temos que a integral fracionária $I_{\mu}$ é comparável em $L_{w}^{p}$ com operador maximal fracionário definido por

$$
M_{\mu} f(x)=\sup _{Q} \frac{1}{|Q|^{1-\frac{\mu}{n}}} \int_{Q}|f(y)| d y,
$$

onde o supremo é tomado sobre todo cubo $Q$ com centro $x$.

Baseados em [W] e [CWW] apresentaremos neste trabalho um estudo sobre desigualdades ponderadas para operadores maximais que generalizam o operador maximal fracionário (0.3), para ser mais preciso, estudaremos basicamente os operadores maximais definidos abaixo:

Dado $0 \leq \mu<n$ e dois conjuntos (possivelmente não relacionados) $S$ e $E$ em $\mathbb{R}^{n}$, definamos o operador maximal não centrado

$$
M_{S, E, \mu} f(x)=\sup _{\substack{z \in \mathbb{R}^{n}, t>0 \\ x \in z+t E}} t^{\mu-n} \int_{z+t S}|f(y)| d y,
$$

onde $z+t E$ denota o conjunto $\{z+t \xi ; \xi \in E\}$ e similarmente $z+t S$.

Definamos também o operador maximal centrado

$$
M_{S, \mu} f(x)=\sup _{t>0} t^{\mu-n} \int_{x+t S}|f(y)| d y, \quad 0 \leq \mu<n,
$$

que corresponde a tomar $E$ vazio em (0.4).

Observemos que para $S=Q_{1}$, onde $Q_{1}$ é o cubo unitário centrado na origem, $M_{S, \mu}$ recai no operador $M_{\mu}$ definido acima.

O operador $M_{S, \mu}$ está relacionado com o seguinte caso particular de (0.2)

$$
I_{\alpha, \beta} f(x)=f * K_{\alpha, \beta}(x),
$$

onde $K_{\alpha, \beta}(x)=|x|^{\alpha-n+1}\left|x_{n}\right|^{\beta-1}$, para $x=\left(x_{1}, \ldots, x_{n}\right),-\beta<\alpha<n-1 \mathrm{e}$ $0<\beta<1$. Obtemos $I_{\alpha, \beta}$ tomando $\mu=\alpha+\beta$ e $\Omega(x)=\left(\frac{|x|}{\left|x_{n}\right|}\right)^{1-\beta}$ em (0.2). 
Se colocarmos $\gamma=(n-1-\alpha) /(1-\beta)$, então $\rho(\theta)=\left|\theta_{n}\right|^{-1 /(\gamma+1)}$, assim $S_{\rho}=\left\{r \theta ; \theta \in S^{n-1}, 0 \leq r<\left|\theta_{n}\right|^{-1 /(\gamma+1)}\right\}$ depende somente de $\gamma$.

A seguir, resumiremos como o trabalho está organizado.

Inicialmente apresentaremos, no capítulo 1, preliminares para este assunto. No capítulo 2, daremos uma introdução ao estudo da teoria de pesos $A_{p}$ introduzida por Muckenhoupt [M] em 1972. Em síntese, definiremos as classes de pesos $A_{p}$ e provaremos o teorema de Muckenhoupt.

Ainda no capítulo 2, provaremos estimativas dos tipos fraco e forte para o operador maximal centrado $(0.5)$ com $S=Q_{1}$, onde $Q_{1}$ é cubo unitário centrado na origem. Tais resultados serão utilizados no capítulo 3 para provar o teorema 3.1 que se refere às estimativas dos tipos fraco e forte para $(0.5)$, onde $S=S_{\rho}$ e $\rho(\theta)=\left|\theta_{n}\right|^{-1 /(\gamma+1)}$. Para apresentarmos os resultados, precisaremos do seguinte: para $a \geq 1$ e $\gamma>0$ fixados, associaremos o operador linear $\delta_{a} x=\left(a x_{1}, \ldots, a x_{n-1}, \bar{a}^{-\gamma} x_{n}\right)$ e os retângulos $R_{a}=\delta_{a} Q_{1}$. Dado um retângulo $R$, denotaremos por $\mathcal{B}(R)$ a coleção de todos transladados e dilatados de $R$, isto é,

$$
\mathcal{B}(R)=\left\{z+t R ; z \in \mathbb{R}^{n}, t>0\right\} .
$$

Para $S=S_{\rho} \operatorname{com} \rho(\theta)=\left|\theta_{n}\right|^{-1 /(\gamma+1)}, 1<p \leq q<\infty$ e para $v$ e $w$ pesos, o teorema 3.1 assegura que se o operador maximal centrado $M_{S, \mu}$ satisfaz a estimativa do tipo fraco

$$
w\left(\left\{x ; M_{S, \mu} f(x)>s\right\}\right) \leq\left(\frac{B\|f\|_{L_{v}^{p}}}{s}\right)^{q},
$$

com $B$ independente de $f$ e $\lambda, \lambda>0$, então existe uma constante $C$ tal que para $a \geq 1$ e $R \in \mathcal{B}\left(R_{a}\right), v, w$ satisfazem

$$
|R|^{\frac{\mu}{n}-1}\left(\int_{R} w d x\right)^{1 / q}\left(\int_{R} v^{-p^{\prime} / p} d x\right)^{1 / p^{\prime}} \leq C B a^{(\gamma-n+1)\left(1-\frac{\mu}{n}\right)},
$$

onde $C$ é uma constante independente de $v, w$ e $a$. Reciprocamente, se existe uma função não crescente $C(a)$ tal que para todo $a \geq 1$ e $R \in \mathcal{B}\left(R_{a}\right)$

$$
|R|^{\frac{\mu}{n}-1}\left(\int_{R} w d x\right)^{1 / q}\left(\int_{R} v^{-p^{\prime} / p}\right)^{1 / p^{\prime}} \leq C(a) a^{(\gamma-n+1)\left(1-\frac{\mu}{n}\right)}
$$

e se $C(a)$ também satisfaz

$$
\int_{1}^{\infty} C(a) \frac{d a}{a}=b
$$


então $M_{S, \mu}$ satisfaz a estimativa do tipo fraco, com $B \leq c b$ para alguma constante $c$ independente de $v, w$ e $f$. Ainda, vale a estimativa do tipo forte

$$
\left\|M_{S, \mu} f\right\|_{L_{w}^{q}} \leq B\|f\|_{L_{v}^{p}}
$$

se existir $r>1$, tal que

$$
|R|^{\frac{\mu}{n}-\frac{1}{p}}\left(\int_{R} w d x\right)^{1 / q}\left(\frac{1}{|R|} \int_{R} v^{-r p^{\prime} / p}\right)^{1 / r p^{i}} \leq C(a) a^{(\gamma-n+1)\left(1-\frac{\mu}{n}\right)},
$$

para todo $R \in \mathcal{B}\left(R_{a}\right)$ e $a \geq 1$, onde $C(a)$ satisfaz (0.7). É claro que, (0.8) é mais forte que (0.6) pela desigualdade de Hölder.

Observemos que as condições acima são semelhantes à condição $A_{p}$.

No capítulo 3 , provaremos o teorema 3.1 citado acima e apresentaremos estimativas dos tipos fraco e forte para o operador maximal não centrado definido em (0.4).

A desigualdade do tipo fraco para o operador não centrado definido em (0.4) para $S=S_{\rho}$ e $E=Q_{1}$ será abordada no teorema 3.10 do capítulo 3. Para descrever isto, seja $\delta_{a}^{*}$ o operador definido por $\delta_{a}^{*}=\left(a x_{1}, \ldots, a x_{n-1}, x_{n}\right)$ para $a \geq 1$, e seja $R_{a}^{*}=\delta_{a}^{*} Q_{1}$. Para referências futuras $R_{a}^{*}$ é o menor retângulo que contém ambos $R_{a}$ e $Q_{1}$. Para cada $R \in \mathcal{B}\left(R_{a}\right)$, associamos um retângulo $R^{*}$ como segue: se $R=z+t R_{a}$, então $R^{*}=z+t R_{a}^{*}$. Provaremos que se $1<p \leq q<\infty \mathrm{e}$

$$
w\left(\left\{x ; M_{S, Q_{1}, \mu} f(x)>s\right\}\right) \leq\left(\frac{B\|f\|_{L_{v}^{p}}}{s}\right)^{q}
$$

então

$$
|R|^{\frac{\mu}{n}-1}\left(\int_{R^{*}} w d x\right)^{1 / q}\left(\int_{R} v^{-p^{\prime} / p} d x\right)^{1 / p^{\prime}} \leq C a^{(\gamma-n+1)\left(1-\frac{\mu}{n}\right)},
$$

para cada $a \geq 1$ e cada par de retângulos $\left(R, R^{*}\right) \in \mathcal{B}\left(R_{a}, R_{a}^{*}\right)$.

Reciprocamente, suponhamos $1<p \leq q<\infty$ e que exista uma função não crescente $C(a)$ tal que para todo $a \geq 1$ e $\left(R, R^{*}\right) \in \mathcal{B}\left(R_{a}, R_{a}^{*}\right)$,

$$
|R|^{\frac{\mu}{n}-1}\left(\int_{R^{*}} w d x\right)^{1 / q}\left(\int_{R} v^{-p^{\prime} / p} d x\right)^{1 / p^{\prime}} \leq C(a) a^{(\gamma-n+1)\left(1-\frac{\mu}{n}\right)} .
$$

Se $C(a)$ também satisfaz (0.7) então $M_{S, Q_{1}, \mu}$ satisfaz a estimativa do tipo fraco (0.9) com $B$ limitada por algum múltiplo fixo de $b$. 
Após as provas dos teoremas 3.1 e 3.10, que são baseadas nos mesmos argumentos, daremos uma versão mais geral do teorema 3.1 , onde consideraremos $S$ um conjunto estrelado em relação a origem. Notemos que, exceto para no máximo um conjunto de medida nula sobre a fronteira, conjuntos $S$ estrelados em relação à origem são sempre da forma $S=S_{\rho}=\{r \theta ; \theta \in$ $\left.S^{n-1}, 0 \leq r<\rho(\theta)\right\}$, onde $\rho$ é denominada função fronteira de $S$.

O objetivo principal no capítulo 3 é o estudo da desigualdade do tipo forte para o operador maximal não centrado $M_{S, E, \mu}$ definido em (0.4), o qual se resume na prova do próximo teorema. Antes de enunciá-lo, descreveremos um conjunto comparável a $S_{\rho}$ para $\rho(\theta)=\left|\theta_{n}\right|^{-1 /(\gamma+1)}$.

Seja $x=\left(x^{\prime}, x_{n}\right)$ com $x^{\prime}=\left(x_{1}, \ldots, x_{n-1}\right)$ e definamos

$$
S_{\gamma}=\left\{x:\left|x_{n}\right| \leq \min \left\{1, \frac{c_{0}}{\left|x^{\prime}\right|^{\gamma}}\right\}, \quad \gamma>0 .\right.
$$

Observemos que $S_{\gamma}$ é comparável a $S_{\rho}=\left\{r \theta, \theta \in S^{n-1}, 0 \leq r<\right.$ $\left.\left|\theta_{n}\right|^{-1 /(\gamma+1)}\right\}=\left\{x ; K_{\alpha, \beta}(x)>1\right\}$, ou seja, existem constantes $\tau$ e $\tau^{\prime}$ positivas, tais que $\tau S_{\gamma} \subseteq S_{\rho} \subseteq \tau^{\prime} S_{\gamma}$. Conseqüentemente, os operadores obtidos pela troca de $S_{\rho}$ por $S_{\gamma}$ são também comparáveis.

Se tomarmos na definição de $M_{S, E, \mu}, S=S_{\gamma}$ e $E=Q_{1}$ e se escolhermos $c_{0}$ tal que $Q_{1} \subset S_{\gamma}$, então a hipótese em (0.4) de que $x \in z+t E$ simplesmente equivale a exigir que $x$ pertença à porção central de $z+t S_{\gamma}$ que se assemelha a um cubo.

Teorema 0.10 Sejam $\gamma>0$ e $S=S_{\gamma}$. Sejam $0 \leq \mu<n, 1<p \leq q<\infty e$ suponhamos que exista $r>1$ tal que

$$
|R|^{\frac{\mu}{n}-\frac{1}{p}} w\left(R^{*}\right)^{\frac{1}{q}}\left(\frac{1}{|R|} \int_{R} v^{\left(-p^{\prime} r\right) / p} d x\right)^{\frac{1}{r p^{\prime}}} \leq C(a)\left|R_{a}\right|^{\frac{\mu}{n}-1}
$$

para todo $R \in \mathcal{B}\left(R_{a}\right)$ e todo $a \geq 1$, onde $C(a)$ é uma função não crescente que satisfaz (0.7). Então,

$$
\left\|M_{S_{\gamma}, Q_{1}, \mu} f\right\|_{L_{w}^{q}} \leq c\|f\|_{L_{v}^{p}} .
$$

$\mathrm{Na}$ verdade, provaremos este resultado para conjuntos estrelados $S$ em relação a origem e para conjuntos limitados $E$, o qual inclui o teorema acima.

Finalmente, daremos um resultado não encontrado nos artigos estudados, que é análogo ao teorema 3.10 para desigualdade do tipo fraco de $M_{S, E, \mu}$, para $S$ estrelado em relação a origem e $E$ limitado. Deixaremos esta parte por último, pelo fato de sua demonstração ser baseada na prova do teorema 0.10 . 


\section{Capítulo 1}

\section{Preliminares}

Neste capítulo apresentaremos algumas definições e alguns resultados que serão úteis para este trabalho.

Definição 1.1 Uma função mensurável, não negativa e localmente integrável $w$ em $\mathbb{R}^{n}$ é chamada peso. Freqüentemente usaremos as seguintes notações: $|E|=\int_{E} d x$ e $w(E)=\int_{E} w(x) d x$.

Trabalharemos no espaço $L^{p}$ com pesos definido abaixo:

Seja $f: E \rightarrow \mathbb{R}$ uma função mensurável, onde $E$ é um subconjunto mensurável de $\mathbb{R}^{n}$. Para $w$ um peso definimos

$$
L_{w}^{p}(E)=\left\{f ;\left(\int_{E}|f|^{p} w d x\right)^{1 / p}<\infty\right\} \quad \text { se } \quad 0<p<\infty
$$

$\mathrm{e}$

$$
L_{w}^{\infty}(E)=\{f ; \inf \{\alpha: w(\{x \in E ;|f(x)|>\alpha\})=0\}<+\infty\} .
$$

Usaremos as notações:

$$
\begin{gathered}
\|f\|_{L_{w}^{p}}(E)=\left(\int_{E}|f|^{p} w d x\right)^{1 / p}, \\
\|f\|_{L_{w}^{\infty}}(E)=\operatorname{supess}_{E} f=\inf \{\alpha: w(\{x \in E ;|f(x)|>\alpha\})=0\} .
\end{gathered}
$$

No caso $E=\mathbb{R}^{n}$, usaremos apenas $\|f\|_{L_{w}^{p}}$.

Definição 1.2 Uma medida de Borel $\mu \mathrm{em} \mathbb{R}^{n}$ é dita ser dobrante se existir uma constante $c>0$ tal que $\mu(2 Q) \leq c \mu(Q)$ para todo cubo $Q \subset \mathbb{R}^{n}$. 
Teorema 1.3 (lema simples de Vitali)Seja $E \subset \mathbb{R}^{n}$, cuja medida exterior de Lebesgue satisfaz $0<|E|_{e}<\infty$. Suponhamos que $E$ é coberto por uma coleção de cubos $\{Q\}$. Então, existe um número finito de cubos disjuntos $Q_{1}, \ldots, Q_{N}$ em $\{Q\}$ e uma constante $\gamma=\gamma(n)>0$ tais que $\sum_{i=1}^{n}\left|Q_{i}\right|>\gamma|E|_{e}$.

A prova do lema Simples de Vitali pode ser encontrada em [WZ].

Observação: $\mathrm{O}$ lema simples de Vitali continua válido para qualquer medida dobrante $\mu$.

Definição 1.4 Um operador definido em funções sobre um espaço de medida $(M, \mathcal{M}, \mu)$ a valores em $(N, \mathcal{N}, \nu)$ é sublinear se

$$
\left|T\left(f_{1}+f_{2}\right)(x)\right| \leq\left|T\left(f_{1}\right)(x)\right|+\left|T\left(f_{2}\right)(x)\right|,
$$

para quase todo $x$.

Definição 1.5 Um operador $T$ é dito do tipo fraco $(p, q)$ se $1 \leq p \leq \infty$, $1 \leq q<\infty$ e se

$$
\nu(\{x ;|T f(x)|>\lambda\}) \leq\left(\frac{c\|f\|_{L_{\mu}^{p}}}{\lambda}\right)^{q},
$$

para todo $\lambda>0, c$ independente de $f$ e $\lambda$.

Definição 1.6 Um operador $T$ é do tipo forte $(p, q)$ se

$$
\|T f\|_{L_{\nu}^{q}} \leq c\|f\|_{L_{\mu}^{p}}
$$

com $c$ independente de $f$.

É fácil vermos que se $T$ é um operador do tipo forte $(p, q)$ então $T$ é do tipo fraco $(p, q)$.

Teorema 1.7 (teorema de Interpolação de Marcinkiewicz) Seja T um operador sublinear que é simultaneamente dos tipos fracos $\left(p_{1}, q_{1}\right)$ e $\left(p_{2}, q_{2}\right)$ com $1 \leq p_{i} \leq q_{i} \leq \infty$, para $i=1,2$ e $q_{1} \neq q_{2}$, então $T$ é do tipo forte $\left(p_{t}, q_{t}\right)$, onde $\frac{1}{p_{t}}=\frac{t}{p_{1}}+\frac{1-t}{p_{2}}$ e $\frac{1}{q_{t}}=\frac{t}{q_{1}}+\frac{1-t}{q_{2}}$, para todo $0<t<1$.

A prova do teorema de interpolação de Marcinkiewicz pode ser encontrada em [T].

Observemos que se $p_{1}=q_{1}$ e $p_{2}=q_{2}$, e $T$ é dos tipos fracos $\left(p_{1}, p_{1}\right)$ e $\left(q_{2}, q_{2}\right)$ então é fácil ver que $p_{t}=q_{t}$, e quando $t$ percorre $(0,1), p_{t}$ cobre todo o intervalo $\left(p_{1}, p_{2}\right)$. 
Para uma medida $\mu$ qualquer definamos

$$
\omega(\alpha)=\omega_{f, E}(\alpha)=\mu(\{x \in E ; f(x)>\alpha\}),
$$

onde $f$ é uma função mensurável sobre $E$ e $-\infty<\alpha<\infty$.

Teorema 1.8 Se $f \geq 0, f \in L_{\mu}^{p}(E)$ então,

$$
\int_{E} f^{p} d \mu=-\int_{0}^{\infty} \alpha^{p} d \omega(\alpha)=p \int_{0}^{\infty} \alpha^{p-1} \omega(\alpha) d \alpha .
$$

A prova deste teorema é análoga à prova com $\mu$ igual à medida de Lebesgue, e esta pode ser encontrada em [WZ].

Teorema 1.9 Seja $f$ uma função mensurável sobre $E$, e seja $E_{a b}=\{x \in$ $E ; a<f(x) \leq b\}$ (a e b finitos). Então,

$$
\int_{E_{a b}} f=-\int_{a}^{b} \alpha d \omega(\alpha)
$$

A prova também pode ser encontrada em [WZ].

Definição 1.10 Dizemos que uma família $K$ de retângulos tem sobreposição limitada se existe uma constante c tal que cada $x \in \mathbb{R}^{n}$ pertença no máximo a c retângulos de $K$. Assim, $K$ tem sobreposição limitada se e somente se,

$$
\sum_{R \in K} \chi_{R}(x) \leq c, \quad x \in \mathbb{R}^{n} .
$$

O próximo teorema é um substituto para o lema simples de Vitali.

Teorema 1.11 (lema de Recobrimento de Besicovitch) Seja E um subconjunto limitado de $\mathbb{R}^{n}$ e seja $K$ uma família de retângulos $R$ cobrindo $E$, tal que $R \in \mathcal{B}\left(R_{0}\right)$, onde $R_{0}$ é um retângulo centrado na origem com lados paralelos aos eixos coordenados. Então, se $R_{x}$ denota um retângulo com centro em $x$ para $x \in E$, existem pontos $\left\{x_{k}\right\}$ em $E$ tal que

(i) $E \subset \cup R_{x_{k}}$,

(ii) $\left\{R_{x_{k}}\right\}$ tem sobreposição limitada.

Ainda mais, a constante c para qual $\sum \chi_{R_{x_{k}}} \leq c$ pode ser escolhida dependendo somente de $n$. 
A prova do lema de Besicovitch "standard"para cubos permanece praticamente a mesma se trocarmos cubos por transladados e dilatados de algum retângulo fixado, sem qualquer mudança nos argumentos sobre a cobertura ou da constante $c$. A prova deste lema para cubos pode ser encontrada em [WZ].

Definição $\mathbf{1 . 1 2} O$ espaço $L_{w}^{q, \infty}$ é definido como sendo a coleção de todas as funçôes mensuráveis $f, f: \mathbb{R}^{n} \rightarrow \mathbb{R}$, tais que $\|f\|_{L_{w}^{q, \infty}}<\infty$, onde

$$
\|f\|_{L_{w}^{q, \infty}}=\sup _{\lambda>0} \lambda\left[w\left(\left\{x \in \mathbb{R}^{n} ;|f(x)|>\lambda\right\}\right)\right]^{1 / q}, 0<q \leq \infty .
$$

Teorema 1.13 espaço $L_{w}^{q, \infty}$ é um espaço de Banach para $1<q \leq \infty$.

A prova deste teorema pode ser encontrada em $[\mathrm{H}]$. 


\section{Capítulo 2}

\section{As Classes de Pesos $A_{p}$ e o teorema de Muckenhoupt}

\subsection{Função Maximal Homogênea de Hardy- Littlewood.}

Definição 2.1 Sejam w um peso e $f \in L_{w}^{\text {loc }}\left(\mathbb{R}^{n}\right)$. Assuma que $w$ é dobrante (isto é, $w(x) d x$ é uma medida dobrante). Definamos

$$
M_{w} f(x)=\sup _{Q} \frac{1}{w(Q)} \int_{Q}|f| w d y
$$

onde o supremo é tomado sobre todos os cubos $Q$ tais que $x \in Q$.

A função $M_{w} f$ é denominada Função Maximal Homogênea de HardyLittlewood de $f$.

Sobre $M_{w} f$ temos o seguinte:

Teorema 2.2 Se wé dobrante então:

(i) $\left\|M_{w} f\right\|_{L_{w}^{p}} \leq c\|f\|_{L_{w}^{p}, 1<p<\infty}$ (desigualdade do tipo forte $(p, p)$ )

(ii) $w\left(\left\{x ; M_{w} f(x)>\lambda\right\}\right) \leq \frac{c}{\lambda}\|f\|_{L_{w}^{1}}$ (desigualdade do tipo fraco $(1,1)$ )

onde as constantes em $(i)$ e (ii) dependem somente de $n$ e $p$. 
Prova: (ii) Fixemos $\lambda>0$. Consideremos $E=\left\{x ; M_{w} f(x)>\lambda\right\}$. É fácil ver que $E$ é um conjunto mensurável ( $E$ é na verdade um conjunto aberto). Para cada $x \in E$ existe um cubo $Q_{x}$ tal que $x \in Q_{x}$ e

$$
\frac{1}{w\left(Q_{x}\right)} \int_{Q_{x}}|f| w d y>\lambda \text {. }
$$

Seja $B_{N}$ a bola de centro 0 e raio $N$. Consideremos $E \cap B_{N}$. Se $x \in E \cap B_{N}$, como $x \in E$, existe $Q_{x}$ tal que $x \in Q_{x}$ e vale (2.3). Pelo lema de Vitali (teorema 1.3) aplicado a $E \cap B_{N}$ e à cobertura $\left\{Q_{x}\right\}_{x \in E \cap B_{N}}$ segue que existem cubos disjuntos $Q_{1}, \ldots, Q_{M}$ e uma constante $\gamma$ positiva tais que

$$
w\left(E \cap B_{N}\right) \leq \frac{1}{\gamma} \sum_{j=1}^{M} w\left(Q_{j}\right)
$$

que combinada com a desigualdade (2.3), implica que

$$
w\left(E \cap B_{N}\right) \leq \frac{1}{\gamma} \lambda^{-1} \sum_{j=1}^{M} \int_{Q_{j}}|f| w d y \leq \frac{1}{\gamma} \lambda^{-1} \int_{E \cap B_{N}}|f| w d y \leq \frac{1}{\gamma} \lambda^{-1}\|f\|_{L_{w}^{1}} .
$$

Fazendo $N \rightarrow \infty$, concluímos a demonstração de $(i i)$.

Notemos que $(i)$ é imediato quando $p=\infty$. Pelo teorema 1.8 temos

$$
\|g\|_{L_{\mu}^{p}(E)}^{p}=p \int_{0}^{\infty} \lambda^{p-1} \mu(\{x \in E ;|g(x)|>\lambda\}) d \lambda .
$$

Logo,

$$
\left\|M_{w} f\right\|_{L_{w}^{p}}^{p}=p \int_{0}^{\infty} \lambda^{p-1} w\left(\left\{x ; M_{w} f(x)>\lambda\right\}\right) d \lambda .
$$

Dado $\lambda>0$, escreva, $f=f_{\lambda}+f^{\lambda}$ onde

$$
\begin{aligned}
& f_{\lambda}(x)=\left\{\begin{array}{lll}
f(x) & \text { se } & |f(x)| \leq \frac{\lambda}{2} \\
0 & \text { se } & |f(x)|>\frac{\lambda}{2}
\end{array}\right. \\
& f^{\lambda}(x)=\left\{\begin{array}{lll}
f(x) & \text { se } & |f(x)|>\frac{\lambda}{2} \\
0 & \text { se } & |f(x)| \leq \frac{\lambda}{2}
\end{array}\right.
\end{aligned}
$$


Observemos que $f_{\lambda} \in L^{\infty}$ e que $M_{w}(f) \leq M_{w}\left(f_{\lambda}\right)+M_{w}\left(f^{\lambda}\right)$. Assim, se $M_{w} f(x)>\lambda$ então ou $M_{w} f_{\lambda}(x)>\frac{\lambda}{2}$ ou $M_{w} f^{\lambda}(x)>\frac{\lambda}{2}$. Logo,

$$
\begin{aligned}
w\left(\left\{x ; M_{w} f(x)>\lambda\right\}\right) & \leq w\left(\left\{x ; M_{w} f_{\lambda}(x)>\frac{\lambda}{2}\right\}\right) \\
& +w\left(\left\{x ; M_{w} f^{\lambda}(x)>\frac{\lambda}{2}\right\}\right)=I+I I, \quad \text { digamos. }
\end{aligned}
$$

Notemos que, como $\left\|f_{\lambda}\right\|_{L^{\infty}} \leq \frac{\lambda}{2}$, então, $M_{w} f_{\lambda}(x) \leq \frac{\lambda}{2}$ e, portanto, $I=0$. Também, observemos que por $(i i)$

$$
I I \leq \frac{c}{\lambda}\left\|f^{\lambda}\right\|_{L_{w}^{1}}=\frac{c}{\lambda} \int_{\mathbb{R}^{n}}\left|f^{\lambda}\right| w d x=\frac{c}{\lambda} \int_{\left\{x ;|f|>\frac{\lambda}{2}\right\}}|f| w d x .
$$

E assim sendo temos que

$$
\begin{aligned}
\left\|M_{w} f\right\|_{L_{w}^{p}}^{p} & \leq p \int_{0}^{\infty} \lambda^{p-1}\left(\frac{c}{\lambda} \int_{\left\{x ;|f|>\frac{\lambda}{2}\right\}}|f| w d x\right) d \lambda \\
& =c p \int_{0}^{\infty} \lambda^{p-2}\left(\int_{\mathbb{R}^{n}}|f| \chi_{\left\{x ;|f|>\frac{\lambda}{2}\right\}} w d x\right) d \lambda \\
& =c p \int_{\mathbb{R}^{n}}|f| w\left(\int_{0}^{\infty} \lambda^{p-2} \chi_{\left\{x ;|f|>\frac{\lambda}{2}\right\}} d \lambda\right) d x \\
& =c p \int_{\mathbb{R}^{n}}|f| w\left(\int_{0}^{2|f|} \lambda^{p-2} d \lambda\right) d x \\
& =\frac{c p}{p-1} 2^{p-1} \int_{\mathbb{R}^{n}}|f|^{p} w d x=c_{n, p}\|f\|_{L_{w}^{p}}^{p},
\end{aligned}
$$

o que demonstra o teorema.

Consideremos $M_{1} f=M f$, onde $M f$ é o operador definido em (0.1). Pelo teorema 2.2 temos

$$
\|M f\|_{L^{p}} \leq c\|f\|_{L^{p}}, \quad 1<p<\infty
$$

$\mathrm{e}$

$$
|\{x ; M f(x)>\lambda\}| \leq \frac{c}{\lambda}\|f\|_{L^{1}}, \quad p=1 .
$$

Neste ponto cabe o seguinte problema: Caracterizar todos os pesos $w$ tais que

$$
\|M f\|_{L_{w}^{p}} \leq c\|f\|_{L_{w}^{p}}, \quad 1<p \leq \infty
$$




$$
w(\{x ; M f(x)>\lambda\}) \leq \frac{c}{\lambda}\|f\|_{L_{w}^{1}}, \quad p=1 .
$$

Para responder o problema acima observemos que se $p>1$

$$
\begin{aligned}
\frac{1}{|Q|} \int_{Q}|f| d y & =\frac{1}{|Q|} \int_{Q}|f| w^{1 / p} w^{-1 / p} d y \\
& \leq \frac{1}{|Q|}\left(\int_{Q} w^{-p^{\prime} / p} d y\right)^{1 / p^{\prime}}\left(\int_{Q}|f|^{p} w d y\right)^{1 / p} \\
& =\frac{1}{|Q|}\left(\int_{Q} w^{-\frac{1}{p-1}} d y\right)^{1 / p^{\prime}}\left(\int_{Q}|f|^{p} w d y\right)^{1 / p} \\
& =\frac{w(Q)^{1 / p}}{|Q|}\left(\frac{1}{w(Q)} \int_{Q}|f|^{p} w d y\right)^{1 / p}\left(\int_{Q} w^{-\frac{1}{p-1}} d y\right)^{1 / p^{\prime}} \\
& =\frac{1}{|Q|}\left(\int_{Q} w d y\right)^{1 / p}\left(\int_{Q} w^{-\frac{1}{p-1}} d y\right)^{1 / p^{\prime}}\left(\frac{1}{w(Q)} \int_{Q}|f|^{p} w d y\right)^{1 / p} \\
\text { e se } p=1 & =\frac{1}{|Q|} \int_{Q}|f| d y \leq \frac{1}{|Q|}\left(\operatorname{supess}_{Q} \frac{1}{w}\right) \int_{Q} f w d y \\
& =\frac{w(Q)}{|Q|}\left(\operatorname{supess} \frac{1}{w}\right) \frac{1}{w(Q)} \int_{Q} f w d y .
\end{aligned}
$$

Tomando o supremo nas desigualdades acima sobre todos os cubos Q tais que $x \in Q$ segue que:

$$
M f(x) \leq \sup _{Q}\left\{\frac{1}{|Q|}\left(\int_{Q} w d y\right)^{1 / p}\left(\int_{Q} w^{-\frac{1}{p-1}} d y\right)^{\frac{p-1}{p}}\right\} M_{w}\left(|f|^{p}\right)^{1 / p}(x)
$$

se $1<p<\infty$ e

$$
M f(x) \leq \sup _{Q}\left\{\frac{1}{|Q|} \int_{Q} w d y \text { supess } \frac{1}{w}\right\} M_{w} f(x), \quad \text { se } p=1 .
$$

Daí a motivação para a definição das classes de pesos $A_{p}$. 


\subsection{As classes de pesos $A_{p}$ e o teorema de Muckenhoupt.}

Nesta seção introduziremos a classe de pesos $A_{p}$, algumas de suas propriedades e provaremos o teorema de Muckenhoupt.

Definição 2.6 Sejam $1 \leq p<\infty e p^{\prime}=\frac{p}{p-1}$. Dizemos que o peso $w$ satisfaz a condição $A_{p}$ se existir uma constante positiva $C$ tal que para todo cubo $Q \subset \mathbb{R}^{n}$

$$
\frac{1}{|Q|}\left(\int_{Q} w\right)^{1 / p}\left(\int_{Q} w^{-\frac{1}{p-1}}\right)^{1 / p^{\prime}} \leq C, \text { se } 1<p<\infty
$$

ou

$$
\frac{1}{|Q|} \int_{Q} w \operatorname{supess}_{Q} \frac{1}{w} \leq C, \quad \text { se } p=1 \text {. }
$$

Notemos que se $w \in A_{p}$ então, segue de (2.4) e (2.5) que

$$
(M f)^{p} \leq C_{w, p} M_{w}\left(|f|^{p}\right) .
$$

Exemplo: $w(x)=|x|^{\alpha}, x \in \mathbb{R}^{n}$, pertence a $A_{p} \Leftrightarrow-n<\alpha<n(p-1)$.

Lema 2.8 Se $1 \leq p \leq q<\infty$ então $A_{p} \subset A_{q}$.

A prova deste lema decorre simplesmente da desigualdade de Hölder.

$O$ próximo lema nos diz que se $p<q$ e $p$ e $q$ estiverem suficientemente próximos, então a recíproca do lema 2.8 é verdadeira, mais precisamente temos:

Lema 2.9 Se $w \in A_{p}$ para algum $1<p<\infty$ então existe $\varepsilon>0$ tal que $w \in A_{p-\varepsilon}$.

A prova do lema 2.9 é mais elaborada e pode ser encontrada em [FZ] ou em $[\mathrm{T}]$.

Teorema 2.10 (Muckenhoupt) Seja $1<p<\infty$. São equivalentes as seguintes afirmações:

(i) $w \in A_{p}$. 
(ii) Existe $c>0$ tal que

$$
w\left(\left\{x \in \mathbb{R}^{n} ; M f(x)>\lambda\right\}\right) \leq \frac{c}{\lambda^{p}}\|f\|_{L_{w}^{p}}^{p},
$$

para todo $\lambda>0$ e toda $f$.

(iii) Existe $c>0$ tal que

$$
\|M f\|_{L_{w}^{p}} \leq c\|f\|_{L_{w}^{p}}
$$

para toda $f$.

Prova: Suponhamos que (iii) seja válida. Então,

$$
w\left(\left\{x \in \mathbb{R}^{n} ; M f(x)>\lambda\right\}\right) \leq \frac{1}{\lambda^{p}} \int[M f]^{p} w \leq \frac{c}{\lambda^{p}} \int|f|^{p} w=\frac{c}{\lambda^{p}}\|f\|_{L_{w}^{p}}^{p} .
$$

Suponhamos agora que a afirmação (ii) seja válida. Fixado um cubo $Q$, definamos $f(x)=\chi_{Q}(x) w(x)^{-\frac{1}{p-1}}$. Observemos que

$$
\|f\|_{L_{w}^{p}}^{p}=\int_{\mathbb{R}^{n}}\left(\chi_{Q} w^{-\frac{1}{p-1}}\right)^{p} w=\int_{Q} w^{-\frac{1}{p-1}} .
$$

Por outro lado, se $x \in Q$,

$$
\begin{aligned}
M f(x) & =\sup _{x \in I} \frac{1}{|I|} \int_{I} \chi_{Q} w^{-\frac{1}{p-1}} \\
& =\sup _{x \in I} \frac{1}{|I|} \int_{I \cap Q} \chi_{Q} w^{-\frac{1}{p-1}} \geq \frac{1}{|Q|} \int_{Q} w^{-\frac{1}{p-1}}
\end{aligned}
$$

Tomemos em (ii) $\lambda=\frac{1}{|Q|} \int_{Q} w^{-\frac{1}{p-1}}$, o qual, por um momento, assumiremos ser finito e positivo. De (2.11) obtemos $Q \subset\{x ; M f(x)>\lambda\}$ e, portanto,

$$
\begin{aligned}
\int_{Q} w & \leq w(\{x ; M f(x)>\lambda\}) \\
& \leq \frac{c}{\lambda^{p}} \int|f|^{p} w=\frac{c}{\left(\frac{1}{|Q|} \int_{Q} w^{-\frac{1}{p-1}}\right)^{p}} \int_{Q} w^{-\frac{1}{p-1}}
\end{aligned}
$$

Desse modo,

$$
\left(\frac{1}{|Q|} \int_{Q} w\right)\left(\frac{1}{|Q|} \int_{Q} w^{-\frac{1}{p-1}}\right)^{p-1} \leq c
$$


isto é, $w \in A_{p}$.

Se $\int_{Q} w^{-\frac{1}{p-1}}=0$ não há nada a ser demonstrado - estamos assumindo $0 . \infty=0$.

Se $\int_{Q} w^{-\frac{1}{p-1}}=\infty$, mostraremos que $w(x)=0$ para quase todo $x \in \mathbb{R}^{n}$. Para cada $\varepsilon>0$ definamos $w_{\varepsilon}=\max \{w, \varepsilon\}$. Então, $w_{\varepsilon} \geq \varepsilon$ e $w_{\varepsilon} \geq w$. Tomemos $f=\chi \chi_{Q} w_{\varepsilon}^{-\frac{1}{p-1}}$ e $\lambda=\frac{1}{|Q|} \int_{Q} w_{\varepsilon}^{-\frac{1}{p-1}}<\infty$. Obtemos

$$
\int_{Q} w \leq \frac{c}{\left(\frac{1}{|Q|} \int_{Q} w_{\varepsilon}^{-\frac{1}{p-1}}\right)^{p}} \int_{Q} w_{\varepsilon}^{-\frac{1}{p-1}}
$$

e, portanto,

$$
\left(\frac{1}{|Q|} \int_{Q} w\right)\left(\frac{1}{|Q|} \int_{Q} w_{\varepsilon}^{-\frac{1}{p-1}}\right)^{p-1} \leq c
$$

Fazendo $\varepsilon \rightarrow 0_{+}$, pelo teorema da convergência monótona, obtemos $\int_{Q} w=0$ e, portanto, $w(x)=0$ para quase todo $x \in Q$. Como $\int_{Q} w^{-\frac{1}{p-1}}=\infty$, então para todo cubo $I \supset Q, \int_{I} w^{-\frac{1}{p-1}}=\infty$. O mesmo argumento acima mostra que $w(x)=0$ para quase todo $x \in I$. Portanto, $w(x)=0$ para quase todo $x \in \mathbb{R}^{n}$.

Finalmente, suponhamos que a afirmação $(i)$ seja válida. Então pelo lema 2.9 existe $\varepsilon>0$, tal que $w \in A_{p-\varepsilon}$ e, obviamente, $w \in A_{p+\varepsilon}$. Assim, por (2.7), $M f(x) \leq c\left[M_{w}|f|^{p-\varepsilon}(x)\right]^{1 /(p-\varepsilon)}$ e $M f(x) \leq c\left[M_{w}|f|^{p+\varepsilon}(x)\right]^{1 /(p+\varepsilon)}$. Assim,

$$
\{x ; M f(x)>\lambda\}=\left\{x ; M f(x)^{p-\varepsilon}>\lambda^{p-\varepsilon}\right\} \subset\left\{x ; M_{w}\left(|f|^{p-\varepsilon}\right)>\lambda^{p-\varepsilon} / c\right\} .
$$

Portanto,

$$
w(\{x ; M f(x)>\lambda\}) \leq w\left(\left\{x ; M_{w}\left(|f|^{p-\varepsilon}\right)>\lambda^{p-\varepsilon} / c\right\}\right) \leq \frac{C}{\lambda^{p-\varepsilon}} \int|f|^{p-\varepsilon} w
$$

e, analogamente,

$$
w(\{x, M f(x)>\lambda\}) \leq \frac{C}{\lambda^{p+\varepsilon}} \int|f|^{p+\varepsilon} w .
$$

Desse modo, a função maximal de Hardy-Littlewood $M f$ satisfaz as condições do tipo fraco $(p-\varepsilon, p-\varepsilon)$ e $(p+\varepsilon, p+\varepsilon)$. Portanto, pelo teorema 1.7, $M f$ satisfaz a condição do tipo forte $(p, p)$,

$$
\|M f\|_{L_{w}^{p}} \leq c\|f\|_{L_{w}^{p}}
$$


que é o que queríamos demonstrar.

No caso de pesos da classe $A_{1}$, temos o seguinte resultado:

Teorema 2.12 (Muckenhoupt). São equivalentes as seguintes afirmações:

(i) $w \in A_{1}$.

(ii) Existe $c>0$ tal que

$$
w\left(\left\{x \in \mathbb{R}^{n} ; M f(x)>\lambda\right\}\right) \leq \frac{c}{\lambda}\|f\|_{L_{w}^{1}}
$$

para todo $\lambda>0$ e toda $f$.

Prova: Suponhamos que (i) seja satisfeita. Então, por (2.7), existe $c>0$ tal que

$$
\{x ; M f(x)>\lambda\} \subset\left\{x ; M_{w} f(x)>\lambda / c\right\} .
$$

Portanto, pela condição $(i i)$ do teorema 2.2

$$
w(\{x ; M f(x)>\lambda\}) \leq w\left(\left\{x ; M_{w} f(x)>\lambda / c\right\}\right) \leq \frac{C}{\lambda} \int|f| w,
$$

que é a condição $(i i)$.

Agora, suponhamos que (ii) seja satisfeita. Dado um cubo $Q$ seja $A=$ $\operatorname{infess}_{Q} w$. Dado $\varepsilon>0$, existe um conjunto mensurável $E \subset Q$ de medida positiva tal que $w \leq A+\varepsilon$ em $E$. Tomemos $f=\frac{1}{w} \chi_{E}$ e $\lambda=\frac{|E|}{|Q|(A+\varepsilon)}$ em (ii). Desse modo, obtemos para todo $x \in Q$

$$
M f(x) \geq \frac{1}{|Q|} \int_{Q}|f|=\frac{1}{|Q|} \int_{E} \frac{1}{w} \geq \frac{|E|}{|Q|(A+\varepsilon)} .
$$

Portanto, utilizando (ii), chegamos a

$$
\int_{Q} w \leq \frac{c}{\frac{|E|}{|Q|(A+\varepsilon)}} \int \chi_{E} \frac{1}{w} w=c(A+\varepsilon)|Q| .
$$

Fazendo $\varepsilon \rightarrow 0_{+}$, obtemos

$$
\frac{1}{|Q|} \int_{Q} w \leq c A=\underset{Q}{\operatorname{infess} w}
$$

que é o que queríamos demonstrar. 


\subsection{Desigualdades ponderadas para o opera- dor maximal centrado $M_{Q_{1}, \mu}$.}

Retomemos agora o operador maximal centrado definido em (0.5)

$$
M_{S, \mu} f(x)=\sup _{t>0} t^{\mu-n} \int_{x+t S}|f(y)| d y, \quad 0 \leq \mu<\tilde{\imath}
$$

Observemos que para $S=Q_{1}$, onde $Q_{1}$ é o cubo unitário centrado na origem:

$$
M_{Q_{1}, \mu} f(x)=\sup _{t>0} t^{\mu-n} \int_{x+t Q_{1}}|f(y)| d y=\sup _{Q}|Q|^{\frac{\mu}{n}-1} \int_{Q}|f(y)| d y,
$$

onde o supremo é tomado sobre todo cubo $Q$ centrado em $x$, uma vez que podemos escrever um dado cubo $Q$ na forma $x+t Q_{1}, t>0$. Observemos também que se $\mu=0, M_{Q_{1}, \mu}$ recai no operador usual $M f$.

Para o operador $M_{Q_{1}, \mu}$, temos os seguintes resultados:

Lema 2.13 Suponhamos que $f$ é uma função não negativa limitada com suporte compacto. Para cada $t>0$, seja $E_{t}=\left\{x \in \mathbb{R}^{n} ; M_{Q_{1}, \mu} f(x)>t\right\}, 0 \leq$ $\mu<n$. Então, se $E_{t}$ é não vazio, temos

$$
E_{t} \subset \bigcup_{j} 3 Q_{j}
$$

onde $Q_{j}$ é a família de cubos diádicos maximais satisfazendo

$$
\frac{t}{4^{n}}<\left|Q_{j}\right|^{\frac{\mu}{n}-1} \int_{Q_{j}} f(y) d y \leq \frac{t}{2^{n}}
$$

para cada $j$.

Ainda mais, temos que

$$
\left\{x \in \mathbb{R}^{n} ; M_{Q_{1}, \mu}^{d} f(x)>\frac{t}{4^{n}}\right\}=\bigcup_{j} Q_{j}
$$

onde $M_{Q_{1}, \mu}^{d}$ denota a correspondente versão diádica de $M_{Q_{1}, \mu}$, ou seja o supremo é tomado sobre todo cubo diádico contendo $x$. 
Prova: Seja $C_{t}=\left\{P_{j}\right\}$ a família de cubos maximais diádicos satisfazendo a condição

$$
t<\left|P_{j}\right|^{\frac{\mu}{n}-1} \int_{P_{j}} f(y) d y
$$

Para mostrar que existe esta tal família $C_{t}$, observemos q11e

$$
|Q|^{\frac{\mu}{n}-1} \int_{Q} f(y) d y \rightarrow 0
$$

$\operatorname{com} Q \uparrow \mathbb{R}^{n}$, pois $f$ tem suporte compacto e $\lim _{t \rightarrow \infty} t^{\frac{\mu}{n}-1}=0$. Se para algum cubo diádico $Q$

$$
t<|Q|^{\frac{\mu}{n}-1} \int_{Q} f(y) d y
$$

então $Q$ está contido num cubo diádico maximal com relação à inclusão satisfazendo (2.16), cuja existência é garantida pela observação (2.15). Assim, existe uma família de cubos diádicos maximais $\left\{P_{j}\right\}$ satisfazendo (2.16). Da maximalidade dos cubos $P_{j}$ resulta

$$
t<\left|P_{j}\right|^{\frac{\mu}{n}-1} \int_{P_{j}} f(y) d y \leq 2^{n}\left|P_{j}^{\prime}\right|^{\frac{\mu}{n}-1} \int_{P_{j}^{\prime}} f(y) d y \leq 2^{n} t
$$

onde $P_{j}^{\prime}$ denota o menor cubo diádico contendo propriamente $P_{j}$. Desta discussão é claro que

$$
\left\{x \in \mathbb{R}^{n} ; M_{Q_{1}, \mu}^{d} f(x)>t\right\}=\bigcup_{j} P_{j} .
$$

Seja $x \in E_{t}$. Pela definição, existe um cubo $R$ contendo $x$ tal que

$$
t<|R|^{\frac{\mu}{n}-1} \int_{R} f(y) d y
$$

Seja $k$ o único inteiro tal que $2^{-(k+1) n}<|R| \leq 2^{-k n}$. Existem cubos diádicos com lados de comprimento $2^{-k}$ e no máximo $2^{n}$ deles, $\left\{J_{i}: i=1, \ldots, 2^{n}\right\}$ tocando o interior de $R$. É fácil ver que, para um destes cubos, digamos $J_{1}$,

$$
\frac{t}{2^{n}}<|R|^{\frac{\mu}{n}-1} \int_{R \cap J_{1}} f(y) d y
$$


Agora, desde que $|R| \leq\left|J_{1}\right|<2^{n}|R|,|R|^{\frac{\mu}{n}} \leq\left|J_{1}\right|^{\frac{\mu}{n}}$, temos

$$
\frac{t}{4^{n}}\left|J_{1}\right|<\frac{t}{2^{n}}|R|<|R|^{\frac{\mu}{n}} \int_{R \cap J_{1}} f(y) d y \leq\left|J_{1}\right|^{\frac{\mu}{n}} \int_{J_{1}} f(y) d y .
$$

Portanto,

$$
\frac{t}{4^{n}}<\left|J_{1}\right|^{\frac{\mu}{n}-1} \int_{J_{1}} f(y) d y .
$$

Pondo $C_{t / 4^{n}}=\left\{Q_{j}\right\}$, vemos que $J_{1} \subset Q_{k}$, para algum $k$, e $R \subset 3 J_{1} \subset 3 Q_{k}$. Disto concluímos que

$$
E_{t} \subset \bigcup_{j} 3 Q_{j}
$$

Finalmente, segue de (2.17) que

$$
\frac{t}{4^{n}}<\left|Q_{j}\right|^{\frac{\mu}{n}-1} \int_{Q_{j}} f(y) d y \leq t 2^{-n},
$$

para cada $j$, e ainda

$$
\left\{x \in \mathbb{R}^{n} ; M_{Q_{1}, \mu}^{d} f(x)>\frac{t}{4^{n}}\right\}=\bigcup_{j} Q_{j} .
$$

Teorema 2.18 Se $1 \leq p \leq q<\infty, 0 \leq \mu<n$, e $v, w$ são pesos, então $M_{Q_{1}, \mu}$ satisfaz a desigualdade do tipo fraco

$$
w\left(\left\{x ; M_{Q_{1}, \mu} f(x)>s\right\}\right) \leq\left(\frac{B_{0}\|f\|_{L_{v}^{p}}}{s}\right)^{q}
$$

se e somente se $v$ e w satisfazem a condição $A_{p, q}^{\mu}$ : isto é, se e somente se existe uma constante $B_{1}$ tal que

$$
\begin{aligned}
& |Q|^{\frac{\mu}{n}-1}\left(\int_{Q} w\right)^{1 / q}\left(\int_{Q} v^{-p^{\prime} / p}\right)^{1 / p^{\prime}} \leq B_{1}, \quad p>1, \\
& |Q|^{\frac{\mu}{n}-1}\left(\int_{Q} w\right)^{1 / q} \operatorname{supess}_{Q}(x)^{-1} \leq B_{1}, \quad p=1,
\end{aligned}
$$

para todo cubo $Q$. As constantes $B_{0}$ e $B_{1}$ são comparáveis, com constantes de comparabilidade independentes de $v$ e $w$. 
Prova: Primeiramente assumiremos que vale (2.20). Para $0<v(x)<\infty$ quase sempre em $Q_{j}$, pelo lema 2.13 e por (2.20) temos:

$$
\begin{aligned}
& w\left(\left\{x ; M_{Q_{1}, \mu} f(x)>s\right\}\right) \leq \sum_{j} \int_{3 Q_{j}} w d x \\
& \leq \sum_{j}\left[\frac{4^{n}\left|Q_{j}\right|^{\frac{\mu}{n}-1}}{s} \int_{Q_{j}} f(y) d y\right]^{q} \int_{3 Q_{j}} w d x \\
& =\frac{4^{n q}}{s^{q}} \sum_{j}\left[\left(\int_{Q_{j}} f v^{1 / p} v^{-1 / p} d y\right)\left(\int_{3 Q_{j}} w d x\right)^{1 / q}\left|Q_{j}\right|^{\frac{\mu}{n}-1}\right]^{q} \\
& \leq \frac{\left(4^{n} 3^{\left(1-\frac{\mu}{n}\right)}\right)^{q}}{s^{q}} \sum_{j}\left[\left(\int_{Q_{j}} f^{p} v d y\right)^{1 / p}\left(\int_{3 Q_{j}} v^{-p^{\prime} / p} d y\right)^{1 / p^{\prime}}\right. \\
& \left.\cdot\left(\int_{3 Q_{j}} w d x\right)^{1 / q}\left|3 Q_{j}\right|^{\frac{\mu}{n}-1}\right]^{q} \text {, por Hölder } \\
& \leq \frac{\overbrace{\left(4^{n} 3^{\left(1-\frac{\mu}{n}\right)} B_{1}\right.}^{B_{0}}}{s^{q}} \sum_{j}\left(\int_{Q_{j}} f^{p} v d y\right)^{q / p} \leq \frac{B_{0}^{q}}{s^{q}}\left(\sum_{j} \int_{Q_{j}} f^{p} v d y\right)^{q / p}, \quad(p \leq q) \\
& =\left(\frac{B_{0}}{s}\left(\int_{\cup_{j}, j} f^{p} v d y\right)^{1 / p}\right)^{q} \leq\left(\frac{B_{0}\|f\|_{L_{v}^{p}}}{s}\right)^{q} \text {. }
\end{aligned}
$$

Se $v(x)=0$ em um subconjunto de medida positiva de $Q_{j}$, por (2.20) $\int_{Q_{j}} w d x=0$. Logo $w(x)=0$ para quase todo $x \in Q_{j}$. Como $\int_{Q_{j}} v^{-1 /(p-1)}=$ $\infty$, então para todo cubo $I \supset Q, \int_{I} v^{-1 /(p-1)}=\infty$. Novamente por $(2.20)$ temos $\int_{I} w d x=0$, logo $w(x)=0$ para quase todo $x \in I$. Portanto, $w(x)=0$ para quase todo $x \in \mathbb{R}^{n}$. 
Suponhamos agora que vale (2.21). Pelo lema 2.13 temos:

$$
\begin{aligned}
& w\left(\left\{x ; M_{Q_{1}, \mu} f(x)>s\right\}\right) \leq \sum_{j} \int_{3 Q_{j}} w d x \\
& \leq \sum_{j}\left(\frac{4^{n}\left|Q_{j}\right|^{\frac{\mu}{n}-1}}{s} \int_{Q_{j}} f d y\right)^{q} \int_{3 Q_{j}} w d x \\
& \leq \frac{4^{n q} 3^{\left(1-\frac{\mu}{n}\right) q}}{s^{q}} \sum_{j}\left[\operatorname{supess}_{3 Q_{j}} v(y)^{-1} \int_{Q_{j}} f v d y\right. \\
& \left.\cdot\left(\int_{3 Q_{j}} w d x\right)^{1 / q}\left|3 Q_{j}\right|^{\frac{\mu}{n}-1}\right]^{q} \\
& \leq \frac{\overbrace{\left(4^{n} 3^{\left(1-\frac{\mu}{n}\right)} B_{1}\right.}^{B_{0}}}{s^{q}}\left(\sum_{j} \int_{Q_{j}} f v d y\right)^{q} \\
& =\frac{B_{0}^{q}}{s^{q}}\left(\int_{\cup_{j} Q_{j}} f v d y\right)^{q} \leq\left(\frac{B_{0}\|f\|_{L_{v}^{1}}}{s}\right)^{q} .
\end{aligned}
$$

Os argumentos para $v(y)=0$ e $v(y)=\infty$ são análogos aos dados acima. Mostramos assim a condição necessária. Para mostrarmos a condição suficiente, suponhamos que vale (2.19). Fixemos um cubo $Q \subset \mathbb{R}^{n}$ e definamos

$$
A=\int_{Q} v^{-p^{\prime} / p} d x
$$

Se $A=0,(2.20)$ vale para qualquer $B_{1}$.

Se $0<A<\infty$, seja $f(x)=\chi_{Q}(x) v(x)^{-p^{\prime} / p}$. Então, para $x \in Q$

$$
\begin{aligned}
M_{Q_{1}, \mu} f(x) & =\sup _{t>0} t^{\mu-n} \int_{x+t Q_{1}} \chi_{Q} v^{-p^{\prime} / p} d y \\
& \geq t^{\mu-n} \int_{Q} v^{-p^{\prime} / p} d y=|Q|^{\frac{\mu}{n}-1} A .
\end{aligned}
$$

Portanto, $Q \subset\left\{x ; M_{Q_{1}, \mu} f(x)>|Q|^{\frac{\mu}{n}-1} A\right\}$, logo por (2.19) temos

$$
\begin{aligned}
\left(\int_{Q} w d x\right)^{1 / q} & \leq B_{0}\left(|Q|^{\frac{\mu}{n}-1} A\right)^{-1}\left(\int_{Q} v^{-p^{\prime}} v d x\right)^{1 / p} \\
& =B_{0}|Q|^{1-\frac{\mu}{n}} A^{-1} A^{1 / p}=B_{0}|Q|^{1-\frac{\mu}{n}} A^{-1 / p^{\prime}}
\end{aligned}
$$


pois $A<\infty, v(x)>0$ quase sempre em $Q$. Multiplicando ambos os lados da desigualdade acima por $|Q|^{\frac{\mu}{n}-1} A^{1 / p^{\prime}}$, obtemos $(2.20)$ com $B_{1} \leq B_{0}$.

Se $A=\infty$, então $(v(x))^{-1 / p}$ não está em $L^{p^{\prime}}$ sobre $Q$. Dessa forma, existe uma função $g(x)$, a qual está em $L^{p}$ sobre $Q$ e que se anula fora de $Q$ tal que $\int_{Q} g(x) v(x)^{-1 / p} d x=\infty$. Definamos $f(x)=g(x) v(x)^{-1 / p}$. Então como $[f(x)]^{p} v(x) \leq[g(x)]^{p},[f(x)]^{p} v(x)$ é integrável sobre $Q$, embora $M_{Q_{1}, \mu} f(x)=$ $\infty$ em quase todo $Q$. Assim (2.19) implica que $\int_{Q} w(x) d x=0, \operatorname{logo}(2.20)$ vale para qualquer $B_{1}$.

Provemos agora que (2.19) implica (2.21).

Se $\operatorname{infess}_{Q} v(y)=\infty$ então (2.21) vale para qualquer $B_{1}$. Caso contrário, dado $\varepsilon>0$, existe um subconjunto $E$ de $Q$ tal que $|E|>0$ e $v(x)<\operatorname{infess}_{Q} v(y)+\varepsilon$ para todo $x \in E$. Definamos $f(x)=1$ sobre $E$ e 0 fora de $E$. Assim, para $x \in Q$

$$
M_{Q_{1}, \mu} f(x)=\sup _{Q}|Q|^{\frac{\mu}{n}-1} \int_{Q}|f| d y=\sup _{Q}|Q|^{\frac{\mu}{n}-1} \int_{E} d y \geq|Q|^{\frac{\mu}{n}-1}|E| .
$$

Logo por (2.19)

$$
\begin{aligned}
\left(\int_{Q} w d x\right)^{1 / q} & \leq B_{0}|Q|^{1-\frac{\mu}{n}}|E|^{-1}\left(\int_{E} v(x) d x\right)^{1 / p} \\
& \leq B_{0}|Q|^{1-\frac{\mu}{n}}|E|^{-1} \int_{E}\left(\operatorname{infess}_{Q} v(y)+\varepsilon\right) d y \\
& =B_{0}|Q|^{1-\frac{\mu}{n}}|E|^{-1}|E|(\underset{Q}{\operatorname{infess} v(y)+\varepsilon)} .
\end{aligned}
$$

Fazendo $\varepsilon \rightarrow 0$, obtemos (2.21).

Lema 2.22 Seja $N$ o operador definido por

$$
N f(x)=\sup _{x \in Q}\left(\frac{1}{|Q|} \int_{Q}|f(y)|^{\left(r p^{\prime}\right)^{\prime}} d y\right)^{1 /\left(r p^{\prime}\right)^{\prime}}
$$

onde $1<p<\infty, p^{\prime}=\frac{p}{p-1}$. Se $r>1$ então:

$$
N: L^{p}\left(\mathbb{R}^{n}\right) \longrightarrow L^{p}\left(\mathbb{R}^{n}\right)
$$

Prova: Observemos que

$$
N f(x)=\left(M(f(y))^{\left(r p^{\prime}\right)^{\prime}}\right)^{1 /\left(r p^{\prime}\right)^{\prime}},
$$


onde $M$ é o operador definido em (0.1).

Se tomarmos $q=\frac{p}{\left(r p^{\prime}\right)^{\prime}}$ temos

$$
\begin{aligned}
q=\frac{p}{\left(r p^{\prime}\right)^{\prime}} & =\frac{p}{\frac{r p^{\prime}}{r p^{\prime}-1}} \\
& =\frac{p\left(r p^{\prime}-1\right)}{r p^{\prime}}=p-\frac{p}{r p^{\prime}} \\
& =p-\frac{1}{r}(p-1) \stackrel{(\mathrm{r}>1)}{>} p-(p-1)=1 .
\end{aligned}
$$

Portanto, como $q>1$, por $(i)$ do teorema $2.2 \operatorname{com} w=1$, segue que

$$
\begin{aligned}
\|N f\|_{L^{p}} & =\left\|\left(M(f(y))^{\left(r p^{\prime}\right)^{\prime}}\right)^{1 /\left(r p^{\prime}\right)^{\prime}}\right\|_{L^{p}} \\
& =\left[\left(\int_{\mathbb{R}^{n}}\left(M|f(y)|^{\left(r p^{\prime}\right)^{\prime}}\right)^{p /\left(r p^{\prime}\right)^{\prime}} d y\right)^{\left(r p^{\prime}\right)^{\prime} / p}\right]^{1 /\left(r p^{\prime}\right)^{\prime}} \\
& \leq\left[c\left(\int_{\mathbb{R}^{n}}\left(|f(y)|^{\left(r p^{\prime}\right)^{\prime}}\right)^{p /\left(r p^{\prime}\right)^{\prime}} d y\right)^{\left(r p^{\prime}\right)^{\prime} / p}\right]^{1 /\left(r p^{\prime}\right)^{\prime}} \\
& \leq c\left(\int_{\mathbb{R}^{n}}|f(y)|^{p} d y\right)^{1 / p}=c\|f\|_{L^{p}} .
\end{aligned}
$$

Teorema 2.23 Sejam $1<p \leq q<\infty, 0<\mu<n$ e suponhamos que para algum $r>1$ que $v$ e w satisfaçam o seguinte fortalecimento da condição $A_{p, q}^{\mu}:$

$$
|Q|^{\mu / n-1 / p}\left(\int_{Q} w d y\right)^{1 / q}\left(\frac{1}{|Q|} \int_{Q} v^{-\frac{r p^{\prime}}{p}} d y\right)^{1 / r p^{\prime}} \leq B_{1}
$$

para todo cubo $Q$. Então $M_{Q_{1}, \mu}$ satisfaz a desigualdade do tipo forte

$$
\left\|M_{Q_{1}, \mu} f(x)\right\|_{L_{w}^{q}} \leq B_{0}\|f\|_{L_{v}^{p}} .
$$

com $B_{0} \leq c B_{1}$, para alguma constante $c$ independente de $v$ e $w$. 
Prova: Para provarmos esse teorema, é suficiente mostrar que existe uma constante $B_{0}$ tal que

$$
\left(\int_{\mathbb{R}^{n}}\left[M_{Q_{1}, \mu} f\right]^{q} w d y\right)^{1 / q} \leq B_{0}\left(\int_{\mathbb{R}^{n}} f^{p} v d y\right)^{1 / p}
$$

para cada função $f$ limitada não negativa com suporte compacto.

Para cada inteiro $k$, e para uma constante $a>2^{n}$ que será escolhida mais adiante, sejam $\Omega_{k}$ e $D_{k}$ os conjuntos

$$
\Omega_{k}=\left\{x \in \mathbb{R}^{n} ; a^{k}<M_{Q_{1}, \mu} f(x)\right\}
$$

$\mathrm{e}$

$$
D_{k}=\left\{x \in \mathbb{R}^{n} ; \frac{a^{k}}{4^{n}}<M_{Q_{1}, \mu}^{d} f(x)\right\} .
$$

Usando o lema 2.13 com $t=4^{n} a^{k}$, existe uma família de cubos maximais diádicos $\left\{Q_{k, j}\right\}$ para qual $\Omega_{k} \subset \cup_{j} 3 Q_{k, j}$ e $D_{k}=\cup_{j} Q_{k, j}$, e

$$
a^{k}<\left|Q_{k, j}\right|^{\frac{\mu}{n}-1} \int_{Q_{k, j}} f(y) d y \leq 2^{n} a^{k}
$$

Com esta notação temos a seguinte seqüência de desigualdades:

$$
\begin{aligned}
& \left(\int_{\mathbb{R}^{n}}\left(M_{Q_{1}, \mu} f(x)\right)^{q} w d y\right)^{1 / q} \\
& =\left(\sum_{k} \int_{\Omega_{k}-\Omega_{k+1}}\left(M_{Q_{1}, \mu} f(x)\right)^{q} w d y\right)^{1 / q} \\
& \leq a\left(\sum_{k} a^{k q} w\left(\Omega_{k}\right)\right)^{1 / q} \leq c\left(\sum_{k, j}\left(\left|Q_{k, j}\right|^{\frac{\mu}{n}-1} \int_{Q_{k, j}} f(y) d y\right)^{q} w\left(3 Q_{k, j}\right)\right)^{1 / q} \\
& \leq C\left(\sum_{k, j}\left(\left|3 Q_{k, j}\right|^{\frac{\mu}{n}-1} \int_{Q_{k, j}} f v^{1 / p} v^{-1 / p} d y\right)^{q} w\left(3 Q_{k, j}\right)\right)^{1 / q}
\end{aligned}
$$




$$
\begin{aligned}
\leq & \left(\sum_{k, j}\left|3 Q_{k, j}\right|^{\frac{\mu}{n}-1}\left(\int_{Q_{k, j}}\left(f v^{1 / p}\right)^{\left(r p^{\prime}\right)^{\prime}} d y\right)^{1 /\left(r p^{\prime}\right)^{\prime}} \cdot\right. \\
& \left.\cdot\left(\int_{Q_{k, j}} v^{-r p^{\prime} / p} d y\right)^{1 / r p^{\prime}} w\left(3 Q_{k, j}\right)^{1 / q}\right)^{q} \\
\leq C & \left(\sum_{k, j}\left|3 Q_{k, j}\right|^{\frac{\mu}{n}}\left(\frac{1}{\left|3 Q_{k, j}\right|} \int_{3 Q_{k, j}}\left(f v^{1 / p}\right)^{\left(r p^{\prime}\right)^{\prime}} d y\right)^{1 /\left(r p^{\prime}\right)^{\prime}} \cdot\right. \\
& \left.\cdot\left(\frac{1}{\left|3 Q_{k, j}\right|} \int_{3 Q_{k, j}} v^{-r p^{\prime} / p} d y\right)^{1 / r p^{\prime}} w\left(3 Q_{k, j}\right)^{1 / q}\right)^{q} \\
\leq C & \left(\sum_{1}\left|3 Q_{k, j}\right|^{1 / p}\left(\frac{1}{\left|3 Q_{k, j}\right|} \int_{3 Q_{k, j}}\left(f v^{1 / p}\right)^{\left(r p^{\prime}\right)^{\prime}} d y\right)^{1 /\left(r p^{\prime}\right)^{\prime}}\right)^{q} \\
\leq & C B_{1}\left(\sum_{k, j}\left[N\left(f v^{1 / p}\right)\right]^{q}\left|3 Q_{k, j}\right|^{q / p}\right)^{1 / q} \\
\leq & \left(\sum_{k, j}\left[N\left(f v^{1 / p}\right)\right]^{p}\left|3 Q_{k, j}\right|\right)^{1 / p}, \text { pois } p \leq q
\end{aligned}
$$

Para cada $k$ seja $D_{k}=\cup_{j} Q_{k, j}$ e seja para cada $k, j$ o conjunto

$$
E_{k, j}=Q_{k, j}-Q_{k, j} \cap D_{k+1}
$$

Então $\left\{E_{k, j}\right\}$ é uma família disjunta de conjuntos que satisfazem com $a>2^{n}$ a estimativa

$$
\left|Q_{k, j}\right|<\frac{1}{1-\frac{2^{n}}{a}}\left|E_{k, j}\right| .
$$


De fato, usando o lema $2.13 \operatorname{com} t=4^{n} a^{k}$ temos

$$
\begin{aligned}
\left|Q_{k, j} \cap D_{k+1}\right| & =\sum_{i}\left|Q_{k, j} \cap Q_{k+1, i}\right|=\sum_{i: Q_{k+1, i} \subset Q_{k, j}}\left|Q_{k+1, i}\right| \\
& <\sum_{i: Q_{k+1, i} \subset Q_{k, j}} \frac{\left|Q_{k+1, i}\right|^{\mu / n}}{a^{k+1}} \int_{Q_{i+1, i}} f(y) d y \\
& \leq \frac{\left|Q_{k, j}\right|^{\mu / n}}{a^{k+1}} \int_{Q_{k, j} \cap\left(\cup_{i} Q_{k+1, i}\right)} f(y) d y \\
& \leq \frac{a^{k} 2^{n}}{a^{k+1}}\left|Q_{k, j}\right|=\frac{2^{n}}{a}\left|Q_{k, j}\right| .
\end{aligned}
$$

e

$$
\begin{aligned}
\frac{\left|E_{k, j}\right|}{\left|Q_{k, j}\right|} & =\frac{\left|Q_{k, j}\right|-\left|Q_{k, j} \cap D_{k+1}\right|}{\left|Q_{k, j}\right|} \\
& =1-\frac{\left|Q_{k, j} \cap D_{k+1}\right|}{\left|Q_{k, j}\right|} \geq 1-\frac{2^{n}}{a}
\end{aligned}
$$

Logo,

$$
\left|Q_{k, j}\right| \leq \frac{1}{1-\frac{2^{n}}{a}}\left|E_{k, j}\right| .
$$

Portanto, concluímos que:

$$
\begin{aligned}
& \left(\int_{\mathbb{R}^{n}}\left(M_{Q_{1}, \mu} f(x)\right)^{q} w d y\right)^{1 / q} \leq C^{\prime} B_{1}\left(\sum_{k, j}\left[N\left(f v^{1 / p}\right)\right]^{p}\left|3 E_{k, j}\right|\right)^{1 / p} \\
& =C^{\prime} B_{1}\left(\sum_{k, j} \int_{3 E_{k, j}}\left[N\left(f v^{1 / p}\right)\right]^{p} d y\right)^{1 / p} \leq C^{\prime} B_{1}\left(\int_{\mathbb{R}^{n}}\left[N\left(f v^{1 / p}\right)\right]^{p} d y\right)^{1 / p} \\
& \leq C^{\prime} B_{1}\left(\int_{\mathbb{R}^{n}}\left(f v^{1 / p}\right)^{p} d y\right)^{1 / p}=C^{\prime} B_{1}\left(\int_{\mathbb{R}^{n}} f^{p} v d y\right)^{1 / p}=B_{0}\|f\|_{L_{v}^{p}}
\end{aligned}
$$

pois, pelo lema 2.22 temos que $N$ é um operador limitado em $L^{p}\left(\mathbb{R}^{n}\right)$. Isto conclui a prova do teorema. 


\section{Capítulo 3}

\section{Desigualdades ponderadas para operadores maximais sobre conjuntos estrelados}

Neste capítulo provaremos o teorema 0.10 da introdução, que se refere à desigualdade do tipo forte para o operador não centrado $M_{S_{\gamma}, Q_{1}, \mu}$. Por motivos de comparação, estudaremos primeiramente as desigualdades tipos fraco e forte para o operador maximal centrado $M_{S, \mu}$. Analisaremos também a desigualdade do tipo fraco para o operador maximal não centrado $M_{S, Q_{1}, \mu}$. Primeiramente consideraremos em ambos os casos $S=S_{\rho}$, onde $S_{\rho}$, é definido abaixo.

Seja $K(x)=K_{\alpha, \beta}(x)=|x|^{\alpha-n+1}\left|x_{n}\right|^{\beta-1}$ para $x=\left(x_{1}, \ldots, x_{n}\right) \operatorname{com}-\beta<$ $\alpha<n-1$ e $0<\beta<1$. Seja $S=S_{\rho}=\left\{r \theta: \theta \in S^{n-1}, 0 \leq r<\rho(\theta)\right\}=$ $\{x ; K(x)>1\}$, onde $\rho(\theta)=\left|\theta_{n}\right|^{-1 /(\gamma+1)}$ com $\gamma=(n-1-\alpha) /(1-\beta)$. É claro que $S$ é um conjunto estrelado com relação à origem.

Posteriormente, consideraremos conjuntos estrelados com relação à origem e conjuntos limitados. Quando dizemos que um conjunto é estrelado, estamos dizendo que o conjunto é estrelado com relação à origem. Vemos que, exceto para no máximo um conjunto de medida nula sobre a fronteira, conjuntos estrelados $S$ são sempre da forma $S=S_{\rho}=\left\{r \theta ; \theta \in S^{n-1}, 0 \leq r<\rho(\theta)\right\}$, e que $S_{\rho}$ é aberto se e somente se $\rho$ é semicontínua inferiormente e $\rho \geq c>0$. Todas as condições sobre $S$ serão feitas em termos da função fronteira $\rho$ de $S$. 


\subsection{Desigualdades dos tipos forte e fraco pa- ra o operador maximal centrado $M_{S, \mu}$ e desigualdade do tipo fraco para o ope- rador maximal não centrado $M_{S, Q_{1}, \mu}$, caso $S=S_{\rho}$.}

Para $a \geq 1$ e $\gamma>0$ fixados, associamos o operador linear $\delta_{a} x=\left(a x_{1}, \ldots\right.$, $\left.a x_{n-1}, a^{-\gamma} x_{n}\right)$ e os retângulos $R_{a}=\delta_{a} Q_{1}$. Dado um retângulo $R$, denotaremos por $\mathcal{B}(R)$ a coleção de todos transladados e dilatados de $R$, isto é,

$$
\mathcal{B}(R)=\left\{z+t R ; z \in \mathbb{R}^{n}, t>0\right\} .
$$

Com esta notação temos

Teorema 3.1 Para $\gamma>0$, seja $S=S_{\rho}$ para $\rho(\theta)=\left|\theta_{n}\right|^{-1 /(\gamma+1)}$. Suponhamos que $1 \leq p \leq q<\infty, 0 \leq \mu<n$, e sejam $v$, w pesos.

(i) Suponhamos que $M_{S, \mu}$ satisfaça a estimativa do tipo fraco

$$
w\left(\left\{x ; M_{S, \mu} f(x)>s\right\}\right) \leq\left(\frac{B\|f\|_{L_{v}^{p}}}{s}\right)^{q} .
$$

Então, existe uma constante $C$ tal que quando $a \geq 1$ e $R \in \mathcal{B}\left(R_{a}\right)$, ve $w$ satisfazem

$$
|R|^{\frac{\mu}{n}-1}\left(\int_{R} w d y\right)^{1 / q}\left(\int_{R} v^{-p^{\prime} / p} d y\right)^{1 / p^{\prime}} \leq C B a^{(\gamma-n+1)\left(1-\frac{\mu}{n}\right)}
$$

$\operatorname{para} p>1$,

$$
|R|^{\frac{\mu}{n}-1}\left(\int_{R} w d y\right)^{1 / q} \operatorname{supess}_{x \in R} v(y)^{-1} \leq C B a^{(\gamma-n+1)\left(1-\frac{\mu}{n}\right)},
$$

para $p=1$. Onde $C$ é uma constante independente de $v, w$ e a.

(ii) Reciprocamente, suponhamos que exista uma função não crescente $C(a)$ tal que para todo $a \geq 1$ e $R \in \mathcal{B}\left(R_{a}\right)$ valha

$$
|R|^{\frac{\mu}{n}-1}\left(\int_{R} w d y\right)^{1 / q}\left(\int_{R} v^{-p^{\prime} / p} d y\right)^{1 / p^{\prime}} \leq C(a) a^{(\gamma-n+1)\left(1-\frac{\mu}{n}\right)}
$$


para $p>1$,

$$
|R|^{\frac{\mu}{n}-1}\left(\int_{R} w d y\right)^{1 / q} \operatorname{supess}_{x \in R} v(y)^{-1} \leq C(a) a^{(\gamma-n+1)\left(1-\frac{\mu}{n}\right)},
$$

para $p=1$. E se C(a) também satisfaz

$$
\int_{1}^{\infty} C(a) \frac{d a}{a}=b, \quad q>1,
$$

$e$

$$
\int_{1}^{\infty} C(a)\left(1+\log ^{+} \frac{1}{C(a)}\right) \frac{d a}{a}=b, \quad p=q=1,
$$

então $M_{S, \mu}$ satisfaz a estimativa do tipo fraco (3.2), com $B \leq$ cb para alguma constante $c$ independente de $v, w$ e $f$.

(iii) Suponhamos que $1<p \leq q<\infty$, e que $v$ e $w$ satisfaçam para algum $r>1$

$$
|R|^{\frac{\mu}{n}-\frac{1}{p}}\left(\int_{R} w d y\right)^{1 / q}\left(\frac{1}{|R|} \int_{R} v^{-r p^{\prime} / p} d y\right)^{1 / r p^{\prime}} \leq C(a) a^{(\gamma-n+1)\left(1-\frac{\mu}{n}\right)},
$$

para todo $R \in \mathcal{B}\left(R_{a}\right)$ e a $\geq 1$, onde $C(a)$ satisfaz (3.7). Então $M_{S, \mu}$ satisfaz a desigualdade do tipo forte

$$
\left\|M_{S, \mu} f\right\|_{L_{w}^{q}} \leq B\|f\|_{L_{v}^{p}} .
$$

A seguir, daremos um resultado para o operador maximal não centrado $M_{s_{\rho}, Q_{1}, \mu}$. Para isto seja $\delta_{a}^{*}$ o operador definido por $\delta_{a}^{*}=\left(a x_{1}, \ldots, a x_{n-1}, x_{n}\right)$ para $a \geq 1$ e seja $R_{a}^{*}=\delta_{a}^{*} Q_{1}$. Para cada $R \in \mathcal{B}\left(R_{a}\right)$, associamos um retângulo $R^{*}$ como segue: se $R=z+t R_{a}$, então $R^{*}=z+t R_{a}^{*}$.

Teorema 3.10 Sejam $S, \gamma, \mu, p$ e q como no teorema 3.1 e sejam $v$ e w pesos.

(i) Suponhamos que $M_{S, Q_{1}, \mu}$ satisfaça a estimativa do tipo fraco

$$
w\left(\left\{x ; M_{S, Q_{1}, \mu} f(x)>s\right\}\right) \leq\left(\frac{B\|f\|_{L_{v}^{p}}}{s}\right)^{q} .
$$


Então, para cada $a \geq 1$ e cada par de retângulos $\left(R, R^{*}\right) \in \mathcal{B}\left(R_{a}, R_{a}^{*}\right)$, $v$ e $w$ satisfazem

$$
|R|^{\frac{\mu}{n}-1}\left(\int_{R^{*}} w d y\right)^{1 / q}\left(\int_{R} v^{-p^{\prime} / p} d y\right)^{1 / p^{\prime}} \leq C a^{(\gamma-n+1)\left(1-\frac{\mu}{n}\right)},
$$

päa $\ddot{p}>1$,

$$
|R|^{\frac{\mu}{n}-1}\left(\int_{R^{*}} w d y\right)^{1 / q} \operatorname{supess}_{R} v(y)^{-1} \leq C a^{(\gamma-n+1)\left(1-\frac{\mu}{n}\right)},
$$

para $p=1$.

Podemos tomar c menor que um múltiplo fixo de $B$ independente de $v, w$ e $a$.

(ii) Reciprocamente, suponhamos que exista uma função não crescente $C(a)$ tal que para todo $a \geq 1, e\left(R, R^{*}\right) \in \mathcal{B}\left(R_{a}, R_{a}^{*}\right)$ valha

$$
|R|^{\frac{\mu}{n}-1}\left(\int_{R^{*}} w d y\right)^{1 / q}\left(\int_{R} v^{-p^{\prime} / p} d y\right)^{1 / p^{\prime}} \leq C(a) a^{(\gamma-n+1)\left(1-\frac{\mu}{n}\right)},
$$

$\operatorname{para} p>1$,

$$
|R|^{\frac{\mu}{n}-1}\left(\int_{R^{*}} w d y\right)^{1 / q} \operatorname{supess}_{R} v(y)^{-1} \leq C(a) a^{(\gamma-n+1)\left(1-\frac{\mu}{n}\right)},
$$

para $p=1$. Se $C\left(\right.$ a) também satisfaz (3.7) e (3.8), então $M_{S, Q_{1}, \mu}$ satisfaz a estimativa do tipo fraco (3.11), com $B$ limitada por algum múltiplo fixo de $b$.

Para provarmos os teoremas 3.1 e 3.10 , precisaremos de alguns resultados: Seja $k(x)=K_{\alpha, \beta}(x)=|x|^{\alpha-n+1}\left|x_{n}\right|^{\beta-1}$ e escrevamos $x \in \mathbb{R}^{n}$ como $x=$ $\left(x^{\prime}, x_{n}\right)$, onde $x^{\prime}=\left(x_{1}, \ldots, x_{n-1}\right)$. Então para $\left|x^{\prime}\right| \geq\left|x_{n}\right|, k(x) \approx\left|x^{\prime}\right|^{\alpha-n+1}$. $\left|x_{n}\right|^{\beta-1}$ e se $\left|x^{\prime}\right| \leq\left|x_{n}\right|, k(x) \approx\left|x_{n}\right|^{\alpha+\beta-n}$. Logo, se $c_{o}>0$ e definirmos

$$
S_{\gamma}=\left\{x ;\left|x_{n}\right| \leq \min \left\{1, c_{o}\left|x^{\prime}\right|^{-\gamma}\right\}\right\}
$$

então $S_{\gamma}$ é comparável a $S=S_{\rho}=\{x ; k(x)>1\}$, ou seja, existem constantes $\tau, \tau^{\prime}>0$ tais que $\tau S_{\gamma} \subseteq S \subseteq \tau^{\prime} S_{\gamma}$. 
Conseqüentemente, os operadores obtidos trocando $S$ por $S_{\gamma}$ são comparáveis.

Fixemos $c_{0}$ para que $Q_{1} \subseteq S_{\gamma}$, o que assegura também que se $a \geq 1$ então o retângulo $R_{a}=\delta_{a} Q_{1}$ está contido em $S_{\gamma}$, pois $\delta_{a}\left(S_{\gamma}\right) \subseteq S_{\gamma}$ para $a \geq 1$. Podemos ver também que $S_{\gamma} \subseteq \cup_{k=0}^{\infty}\left(\kappa R_{2^{k}}\right)$ para uma constante $\kappa$ dependendo somente de $n$ e $c_{0}$. Isto nos dá as seguintes comparações:

$$
\begin{gathered}
\chi_{R_{a}}(x / \tau) \leq \chi_{S}(x), \\
\chi_{S}(x) \leq \sum_{k=0}^{\infty} \chi_{R_{2^{k}}}\left(x /\left(\tau^{\prime} \kappa\right)\right) .
\end{gathered}
$$

Agora,

$$
\begin{aligned}
M_{S, \mu} f(x) & =\sup _{t>0} t^{\mu-n} \int_{x+t S}|f(y)| d y \\
& =\sup _{t>0} t^{\mu-n} \int_{\mathbb{R}^{n}}\left|\chi_{x+t S}(y) f(y)\right| d y \\
& =\sup _{t>0} t^{\mu-n} \int_{\mathbb{R}^{n}}\left|\chi_{t S}(y-x) f(y)\right| d y \\
& \geq \sup _{t>0} t^{\mu-n} \int_{\mathbb{R}^{n}}\left|\chi_{t R_{a}}((y-x) / \tau) f(y)\right| d y, \quad \text { por }(3.16) \\
& =\sup _{t>0} t^{\mu-n} \int_{\mathbb{R}^{n}}\left|\chi_{x+\tau t R_{a}}(y) f(y)\right| d y=\sup _{t>0} t^{\mu-n} \int_{x+\tau t R_{a}}|f(y)| d y \\
& =\sup _{s>0} \tau^{n-\mu} s^{\mu-n} \int_{x+s R_{a}}|f(y)| d y=\tau^{n-\mu} M_{R_{a}, \mu} f(x)
\end{aligned}
$$

Logo,

$$
M_{S, \mu} f(x) \geq c M_{R_{a}, \mu} f(x), \quad a \geq 1
$$

onde $c=\tau^{n-\mu}$.

Analogamente, usando (3.17) temos

$$
M_{S, \mu} f(x) \leq C \sum_{k=0}^{\infty} M_{R_{2^{k}}, \mu} f(x) .
$$

onde $C=\left(\tau^{\prime} \kappa\right)^{n-\mu}$. 
Quando $\Lambda$ for uma transformação linear invertível em $\mathbb{R}^{n}$, identificaremos $\Lambda$ com o operador

$$
\Lambda f(x)=|\operatorname{det} \Lambda| f(\Lambda x)
$$

Observemos que

$$
\Lambda^{-1} M_{S, \mu} \Lambda f=|\operatorname{det} \Lambda|^{-1} M_{\Lambda S, \mu} f
$$

Portanto, se identificarmos $\delta_{a}$ com o operador em (3.20), temos

$$
M_{R_{a}, \mu} f(x)=a^{n-1-\gamma} \delta_{a}^{-1} M_{Q_{1}, \mu} \delta_{a} f(x) .
$$

Para qualquer operador sublinear $T$, qualquer transformação linear invertível $\Lambda$ e quaisquer $p, q>1$, a desigualdade do tipo fraco

$$
w\left(\left\{x:\left|\left(\Lambda^{-1} T \Lambda\right) f(x)\right|>s\right\}\right) \leq\left(\frac{B\|f\|_{L_{v}^{p}}}{s}\right)^{q}
$$

para toda $f \in L^{p}(v)$ e $s>0$ é equivalente à desigualdade

$$
(\Lambda w)(\{x:|T f(x)|>s\}) \leq\left(\frac{B\|f\|_{L_{\Lambda v}^{p}}}{s}\right)^{q},
$$

para toda $f \in L^{p}(\Lambda v)$ e $s>0$, com mesma constante $B$.

De fato: Observemos primeiro que

$$
\begin{aligned}
\Lambda^{-1} T \Lambda f(x) & =|\operatorname{det} \Lambda|^{-1} T \Lambda f\left(\Lambda^{-1} x\right) \\
& =|\operatorname{det} \Lambda|^{-1} T\left(|\operatorname{det} \Lambda| f\left(\Lambda \Lambda^{-1} x\right)\right) \\
& =|\operatorname{det} \Lambda|^{-1}|\operatorname{det} \Lambda| T f(x)=T f(x) .
\end{aligned}
$$

Portanto, $\left\{x ;\left|\left(\Lambda^{-1} T \Lambda\right) f(x)\right|>s\right\}=\{x ;|T f(x)|>s\}=C_{f}$ e

$$
\begin{aligned}
\Lambda C_{f}=\left\{\Lambda x ; x \in C_{f}\right\} & =\{\Lambda x ;|T f(x)|>s\} \\
& =\left\{\Lambda x ;\left|\Lambda^{-1} T \Lambda f(x)\right|>s\right\} \\
& =\left\{y ;\left|\Lambda^{-1} T \Lambda f\left(\Lambda^{-1} y\right)\right|>s\right\} \\
& =\left\{y ;\left|\Lambda^{-1} T \Lambda g(y)\right|>s\right\}=C_{g}
\end{aligned}
$$


onde $y=\Lambda x$ e $g=f \Lambda^{-1}$.

Observemos também que

$$
\begin{aligned}
\Lambda w\left(C_{f}\right)=\int_{C_{f}} \Lambda w(y) d y & =\int_{C_{f}}|\operatorname{det} \Lambda| w(\Lambda y) d y \\
& =\int_{\Lambda C_{f}}|\operatorname{det} \Lambda| w(y) \mid \operatorname{det} \Lambda_{\mid}^{-1} d y \\
& =\int_{\Lambda C_{f}} w(y) d y=w\left(\Lambda C_{f}\right) .
\end{aligned}
$$

Com estes resultados e por (3.23) temos

$$
\begin{aligned}
\Lambda w\left(C_{f}\right) & =w\left(\Lambda C_{f}\right)=w\left(C_{g}\right) \leq\left(\frac{B\|g\|_{L_{v}^{p}}}{s}\right)^{q} \\
& =\left(\frac{B}{s}\left(\int_{\mathbb{R}^{n}}\left|f\left(\Lambda^{-1} x\right)\right|^{p} v(x) d x\right)^{1 / p}\right)^{q} \\
& \left.=\left(\frac{B}{s} \int_{\mathbb{R}^{n}}|f(y)|^{p} v(\Lambda y)|\operatorname{det} \Lambda| d y\right)^{1 / p}\right)^{q} \\
& =\left(\frac{B}{s}\left(\int_{\mathbb{R}^{n}}|f(y)|^{p} \Lambda v(y) d y\right)^{1 / p}\right)^{q}=\left(\frac{B}{s}\|f\|_{L_{\Lambda v}^{p}}\right)^{q},
\end{aligned}
$$

para $y=\Lambda^{-1} x$.

Por outro lado, assumindo (3.24) temos

$$
\begin{aligned}
w\left(C_{f}\right) & =\Lambda^{-1} w\left(\Lambda C_{f}\right)=\Lambda^{-1} w\left(C_{g}\right) \\
& \leq\left(\frac{B\|g\|_{L_{\Lambda-1}^{p}}}{s}\right)^{q}=\left(\frac{B}{s}\left(\int_{\mathbb{R}^{n}}\left|f\left(\Lambda^{-1} x\right)\right|^{p} \Lambda^{-1} v(x) d x\right)^{1 / p}\right)^{q} \\
& =\left(\frac{B}{s}\left(\int_{\mathbb{R}^{n}}|f(y)|^{p}|\operatorname{det} \Lambda|^{-1} v(y)|\operatorname{det} \Lambda| d y\right)^{1 / p}\right)^{q} \\
& =\left(\frac{B}{s}\left(\int_{\mathbb{R}^{n}}|f(y)|^{p} v(y) d y\right)^{1 / p}\right)^{q}=\left(\frac{B\|f\|_{L_{v}^{p}}}{s}\right)^{q},
\end{aligned}
$$

para $y=\Lambda^{-1} x$.

Similarmente a desigualdade

$$
\left\|\Lambda^{-1} T \Lambda f\right\|_{L_{w}^{q}} \leq B^{\prime}\|f\|_{L_{v}^{p}},
$$


é equivalente à desigualdade

$$
\|T f\|_{L_{\Lambda w}^{q}} \leq B^{\prime}\|f\|_{L_{\Lambda v}^{p}}
$$

Assim, por exemplo, vemos de (3.22) que qualquer desigualdade do tipo fraco para $M_{R_{a}, \mu}$ é equivalente neste sentido à desigualdade do tipo fraco para $M_{Q_{1}, \mu}$ com uma constantc apropriada. Então por $(3.18)$ c (3.22) temos

$$
M_{S, \mu} f(x) \geq c a^{-(\gamma-n+1)} \delta_{a}^{-1} M_{Q_{1}, \mu} \delta_{a} f(x), \quad \text { para } a \geq 1
$$

e por (3.19),

$$
M_{S, \mu} f(x) \leq C \sum_{k=0}^{\infty} 2^{-k(\gamma-n+1)} \delta_{2^{-k}} M_{Q_{1}, \mu} \delta_{2^{k}} f(x) .
$$

Para o operador maximal $M_{S, E, \mu}$, observemos que se $S$ e $S^{\prime}$ são conjuntos comparáveis, e se $E$ e $E^{\prime}$ são também conjuntos comparáveis, então $M_{S, E, \mu}$ e $M_{S^{\prime}, E^{\prime}, \mu}$ são comparáveis. Assim, de (3.16) e (3.17) podemos escrever

$$
M_{S, Q_{1}, \mu} f(x) \geq c M_{R_{a}, Q_{1}, \mu} f(x), \quad a \geq 1
$$

$\mathrm{e}$

$$
M_{S, Q_{1}, \mu} f(x) \leq C \sum_{k=0}^{\infty} M_{R_{2^{k}}, Q_{1}, \mu} f(x) .
$$

Precisaremos também do seguinte lema, obtido de [StWe].

Lema 3.31 Suponhamos que para $j=1,2, \ldots, g_{j}(x)$ seja uma função não negativa mensurável para qual $\left|\left\{x ; g_{j}(x)>s\right\}\right|<1 / s$. Seja $\left\{c_{j}\right\}$ uma seqüência de números positivos com $\sum_{j} c_{j}=1$ e colocamos $K=\sum_{j} c_{j} \log \left(1 / c_{j}\right)$. Então, $\left|\left\{x ; \sum_{j} c_{j} g_{j}(x)>s\right\}\right|<2(K+2) / s$.

Prova: Definamos

$$
\begin{gathered}
\ell_{j}(x)=\left\{\begin{array}{lll}
g_{j}(x) & \text { se } & g_{j}(x)<s / 2 \\
0 & \text { se } & g_{j}(x) \geq s / 2 .
\end{array}\right. \\
u_{j}(x)=\left\{\begin{array}{lll}
g_{j}(x) & \text { se } & g_{j}(x)>s / 2 c_{j} \\
0 & \text { se } & g_{j}(x) \leq s / 2 c_{j} .
\end{array}\right.
\end{gathered}
$$


e

$$
m_{j}(x)=g_{j}(x)-\ell_{j}(x)-u_{j}(x)
$$

Então,

$$
\ell(x)=\sum c_{j} \ell_{j}(x)=\left\{\begin{array}{lll}
\sum c_{j} g_{j}(x) & \text { se } & g_{j}(x)<s / 2 \\
0 & \text { se } & g_{j}(x) \geq s / 2 .
\end{array}\right.
$$

Daí $\ell(x)<s / 2$ em quase toda parte, pois $\left|\left\{x ; g_{j}(x)>s\right\}\right|<1 / s$ e $\sum_{j} c_{j}=1$. E

$$
u(x)=\sum c_{j} u_{j}(x)=\left\{\begin{array}{lll}
\sum c_{j} g_{j}(x) & \text { se } & g_{j}(x)>s / 2 c_{j} \\
0 & \text { se } & g_{j}(x) \leq s / 2 c_{j}
\end{array}\right.
$$

Assim $u(x)=0$ exceto em um conjunto de medida menor que $2 / s$ pelos mesmos argumentos acima. Observemos que para

$$
\begin{array}{rlll} 
& g_{j}(x)<s / 2 \leq s / 2 c_{j}, \quad \text { temos } & m_{j}(x)=0 ; \\
& g_{j}(x)>s / 2 c_{j} \geq s / 2, \quad \text { temos } & m_{j}(x)=0 ; \\
\text { e } \quad s / 2 \leq g_{j}(x) \leq s / 2 c_{j}, \quad \text { temos } & m_{j}(x)=g_{j}(x) .
\end{array}
$$

Pondo $\lambda_{j}(y)=\left|\left\{x ; g_{j}(x)>y\right\}\right|, y>0$ e $m(x)=\sum c_{j} m_{j}(x)$ temos

$$
\begin{aligned}
\int m(x) d x & =\int \sum c_{j} m_{j}(x) d x=\sum c_{j} \int m_{j}(x) d x \\
& =\sum c_{j} \int_{\left\{x ; s / 2 \leq g_{j}(x) \leq s / 2 c_{j}\right\}} g_{j}(x) d x \\
& =-\sum c_{j} \int_{s / 2}^{s / 2 c_{j}} y d \lambda_{j}(y), \quad \text { pelo teorema } 1.9 \\
& =\sum c_{j}\left[\int_{s / 2}^{s / 2 c_{j}} \lambda_{j}(y) d y-\left.y \lambda_{j}(y)\right|_{s / 2} ^{s / 2 c_{j}}\right] \\
& <\sum c_{j}\left[\int_{s / 2}^{s / 2 c_{j}} y^{-1} d y+1\right]=K+1
\end{aligned}
$$

e

$$
\begin{aligned}
|\{x ; m(x)>s / 2\}| & =\int_{\{x ; m(x)>s / 2\}} d x=2 / s \int_{\{x ; m(x)>s / 2\}} s / 2 d x \\
& <2 / s \int m(x) d x<2(K+1) / s
\end{aligned}
$$


Sejam

$$
A=\{x ; \ell(x) \geq s / 2\} \cup\{x ; u(x) \neq 0\}
$$

$\mathrm{e}$

$$
C_{g}=\left\{x ; \sum c_{j} g_{j}(x)>s\right\}
$$

Observemos que

$$
|A| \leq|\{x ; \ell(x) \geq s / 2\}|+|\{x ; u(x) \neq 0\}|<2 / s
$$

e que se $x \notin A, u(x)=0$ e $\ell(x)<s / 2$. Então, para $x \notin A$ e $x \in C_{g}$

$$
m(x)=\sum c_{j} g_{j}(x)-\sum c_{j} \ell_{j}(x)-\sum c_{j} u_{j}(x)>s-s / 2=s / 2 .
$$

Portanto,

$\left|C_{g}\right|=\left|C_{g} \cap A\right|+\left|C_{g} \cap A^{c}\right| \leq|A|+2(K+1) / s<2 / s+2(K+1) / s=2(K+2) / s$.

Prova do teorema 3.1:

Parte $(i)$ : Suponhamos que valha a desigualdade do tipo fraco (3.2), logo por (3.27) temos

$$
w\left(\left\{x ; \delta_{a}^{-1} M_{Q_{1}, \mu} \delta_{a} f(x)>s\right\}\right) \leq\left(\frac{c B a^{\gamma-n+1}\|f\|_{L_{v}^{p}}}{s}\right)^{q},
$$

ou pela equivalência entre (3.23) e (3.24),

$$
\delta_{a} w\left(\left\{x ; M_{Q_{1}, \mu} f(x)>s\right\}\right) \leq\left(\frac{c B a^{\gamma-n+1}\|f\|_{L_{\delta_{a} v}^{p}}}{s}\right)^{q} .
$$

Assim, pelo teorema 2.18, obtemos

$$
\begin{gathered}
|Q|^{\frac{\mu}{n}-1}\left(\int_{Q} \delta_{a} w\right)^{1 / q}\left(\int_{Q}\left(\delta_{a} v\right)^{-p^{\prime} / p}\right)^{1 / p^{\prime}} \leq C B a^{\gamma-n+1}, \quad p>1, \\
|Q|^{\frac{\mu}{n}-1}\left(\int_{Q} \delta_{a} w\right)^{1 / q} \underset{Q}{\operatorname{supess}\left(\delta_{a} v\right)^{-1} \leq C B a^{\gamma-n+1}, \quad p=1 .}
\end{gathered}
$$

Para cada cubo $Q=z+t Q_{1}$ existe um único $R \in \mathcal{B}\left(R_{a}\right)$ tal que $R=z+t R_{a}$, portanto se tomarmos a mudança de variáveis $T(x)=z+t \delta_{a}^{-1} x$ para $x \in R_{a}$, 
vemos que estas duas últimas desigualdades são respectivamente equivalentes a (3.3) e (3.4).

Parte (ii): Para $q>1, L_{w}^{q, \infty}$ é um espaço de Banach (teorema 1.13), assim por (3.28)

$$
\begin{aligned}
\left\|M_{s, \mu} f\right\|_{L_{w}^{q, \infty}}^{q} & =\sup _{\lambda>0}\left(\lambda^{q} w\left(\left\{x \in \mathbb{R}^{n} ; M_{S, \mu} f(x)>\lambda\right\}\right)\right) \\
& \leq \sup _{\lambda>0}\left(\lambda^{q} w\left(\left\{x \in \mathbb{R}^{n} ; C \sum_{k=0}^{\infty} 2^{-k(\gamma-n+1)} \delta_{2^{-k}} M_{Q_{1}, \mu} \delta_{2^{k}} f(x)>\lambda\right\}\right)\right) \\
& \leq C \sum_{k=0}^{\infty} 2^{-k(\gamma-n+1)} \sup _{\lambda>0}\left(\lambda^{q} w\left(\left\{x \in \mathbb{R}^{n} ; \delta_{2^{-k}} M_{Q_{1}, \mu} \delta_{2^{k}} f(x)>\lambda\right\}\right)\right) \\
& =C \sum_{k=0}^{\infty} 2^{-k(\gamma-n+1)}\left\|\delta_{2^{-k}} M_{Q_{1}, \mu} \delta_{2^{k}}\right\|_{L_{w}^{q, \infty}}^{q} .
\end{aligned}
$$

Assumindo que valem (3.5) e (3.6), pela mudança de variáveis $T(x)=$ $z+t \delta_{a} x$ para $x \in Q_{1}$, obtemos

$$
|Q|^{\frac{\mu}{n}-1}\left(\int_{Q} \delta_{a} w\right)^{1 / q}\left(\int_{Q}\left(\delta_{a} v\right)^{-p^{\prime} / p}\right)^{1 / p^{\prime}} \leq C(a) a^{\gamma-n+1}, \quad p>1
$$

e

$$
|Q|^{\frac{\mu}{n}-1}\left(\int_{Q} \delta_{a} w\right)^{1 / q} \operatorname{supess}_{Q}\left(\delta_{a} v\right)^{-1} \leq C(a) a^{\gamma-n+1}, \quad p=1 .
$$

Usando agora o teorema 2.18 temos

$$
\delta_{a} w\left(\left\{x ; M_{Q_{1}, \mu} f(x)>s\right\}\right) \leq\left(\frac{c C(a) a^{\gamma-n+1}\|f\|_{L_{\delta_{a} v}^{p}}}{s}\right)^{q},
$$

qual, pela equivalência entre (3.23) e (3.24) é equivalente a

$$
w\left(\left\{x ; \delta_{a}^{-1} M_{Q_{1}, \mu} \delta_{a} f(x)>s\right\}\right) \leq\left(\frac{c C(a) a^{\gamma-n+1}\|f\|_{L_{v}^{p}}}{s}\right)^{q},
$$

Portanto,

$$
\left\|\delta_{2^{-k}} M_{Q_{1}, \mu} \delta_{2^{k}} f\right\|_{L_{w}^{q, \infty}}^{q} \leq\left(c 2^{k(\gamma-n+1)} C\left(2^{k}\right)\|f\|_{\left.L_{v}^{p}\right)^{q}} .\right.
$$


Usando (3.34) na estimativa (3.32) dada acima temos

$$
\left\|M_{S, \mu}\right\|_{L_{w}^{q, \infty}}^{q} \leq C^{\prime} \sum_{k=0}^{\infty} C\left(2^{k}\right)\|f\|_{L_{v}^{p} .}
$$

Por (3.7) a série $\sum_{k=0}^{\infty} C\left(2^{k}\right)$, converge, o que conclui a parte $(i i)$ para $q>1$. Para $q=1$, usaremos o lema 3.31 .

Tomemos no lema 3.31

$$
g_{j}(x)=\frac{\delta_{2^{-j}} M_{Q_{1}, \mu} \delta_{2^{j}} f(x)}{c C\left(2^{j}\right) 2^{j(\gamma-n+1)}}
$$

onde $c$ é a constante em (3.33) e

$$
C_{j}=\frac{C\left(2^{j}\right)}{\sum_{k} C\left(2^{k}\right)} .
$$

Para simplificar a notação, como $\sum_{j} C\left(2^{j}\right)$ converge, seja $c^{\prime}$ seu limite. Assumiremos $\|f\|_{L_{v}^{p}}=1$, sem perda de generalidade.

Por (3.33), temos que

$$
\begin{aligned}
w\left(\left\{x ; g_{j}(x)>s\right\}\right) & =w\left(\left\{x ; \delta_{2^{-j}} M_{Q_{1}, \mu} \delta_{2^{j}} f(x)>s c C\left(2^{j}\right) 2^{j(\gamma-n+1)}\right\}\right) \\
& \leq \frac{c C\left(2^{j}\right) 2^{j(\gamma-n+1)}\|f\|_{L_{v}^{p}}}{s c C\left(2^{j}\right) 2^{j(\gamma-n+1)}}=\frac{1}{s}
\end{aligned}
$$

$\mathrm{e}$

$$
\sum_{j} C_{j}=\sum_{j} \frac{C\left(2^{j}\right)}{c^{\prime}}=1 .
$$

Portanto, estão satisfeitas as condições do lema 3.31 e como nesse mesmo lema coloquemos.

$$
K=\frac{1}{c^{\prime}} \sum_{j} C\left(2^{j}\right) \log \left(\frac{c^{\prime}}{C\left(2^{j}\right)}\right) .
$$

Então, pelo lema 3.31 e por (3.28) temos

$$
\begin{aligned}
w\left(\left\{x ; M_{S, \mu} f(x)>s\right\}\right) & \leq w\left(\left\{x ; C \sum_{k=0}^{\infty} 2^{-k(\gamma-n+1)} \delta_{2^{-k}} M_{Q_{1}, \mu} \delta_{2^{k}} f(x)>s\right\}\right) \\
& =w\left(\left\{x ; c c^{\prime} C \sum_{j=1}^{\infty} C_{j} g_{j}(x)>s\right\}\right),
\end{aligned}
$$


onde $j=k+1$ e $C$ é a constante em (3.28). Então, se $\lambda=c c^{\prime} C s$ temos

$$
\begin{aligned}
w\left(\left\{x ; M_{S, \mu} f(x)>\lambda\right\}\right) & \leq w\left(\left\{x ; \sum_{j=1}^{\infty} C_{j} g_{j}(x)>s\right\}\right) \\
& \leq \frac{2(K+2)}{s}=\frac{c c^{\prime} C 2(K+2)}{\lambda}=\frac{c c^{\prime} C 2(K+2)}{\lambda}\|f\|_{L_{v}^{p} .}
\end{aligned}
$$

Para concluir resta mostrar que $K<\infty$. Para isto, observemos que a função contínua

$$
g(x)=\frac{1+\log ^{+} x}{x}=\left\{\begin{array}{lll}
\frac{1+\log x}{x} & \text { se } & x \geq 1 \\
\frac{1}{x} & \text { se } & 0<x \leq 1
\end{array}\right.
$$

é tal que

$$
g^{\prime}(x)=\left\{\begin{array}{llc}
\frac{-\log x}{x^{2}}<0 & \text { se } & x>1 \\
\frac{-1}{x^{2}}<0 & \text { se } & 0<x<1,
\end{array}\right.
$$

ou seja, $g$ é decrescente.

Agora como $C(a)$ é não-crescente, para $a<b$ temos, $C(a) \geq C(b)$ e $0<1 / C(a) \leq 1 / C(b)$. Portanto como $g$ é decrescente temos

$$
g(1 / C(a))=C(a)\left(1+\log ^{+} 1 / C(a)\right) \geq C(b)\left(1+\log ^{+} 1 / C(b)\right)=g(1 / C(b))
$$

e como $1 / a>1 / b$ temos

$$
\frac{C(a)\left(1+\log ^{+} 1 / C(a)\right)}{a} \geq \frac{C(b)\left(1+\log ^{+} 1 / C(b)\right)}{b}
$$

Concluímos assim que a função

$$
f(a)=\frac{C(a)\left(1+\log ^{+} 1 / C(a)\right)}{a}
$$

é não-crescente. Aplicando agora o teste da integral e por (3.8) obtemos

$$
\sum_{j=1}^{\infty} C\left(2^{j}\right)\left(1+\log ^{+} 1 / C\left(2^{j}\right)\right)<\infty
$$

Observemos que

$$
\begin{aligned}
& \int_{1}^{\infty} \frac{C(a)\left(1+\log ^{+} c^{\prime} / C(a)\right)}{a} d a=\int_{1}^{\infty} \frac{C(a)\left(1+\log ^{+} 1 / C(a)+\log ^{+} c^{\prime}\right)}{a} d a \\
& =\int_{1}^{\infty} \frac{C(a)\left(1+\log ^{+} 1 / C(a)\right)}{a} d a+\log ^{+} c^{\prime} \int_{1}^{\infty} \frac{C(a)}{a} d a<\infty
\end{aligned}
$$


por (3.7) e (3.8). Portanto, analogamente obtemos

$$
\sum_{j=1}^{\infty} C\left(2^{j}\right)\left(1+\log ^{+} c^{\prime} / C\left(2^{j}\right)\right)<\infty
$$

E, como

$$
\sum_{j=1}^{\infty} C\left(2^{j}\right) \log \left(\frac{c^{\prime}}{C\left(2^{j}\right)}\right) \leq \sum_{j=1}^{\infty} C\left(2^{j}\right)\left(1+\log ^{+} c^{\prime} / C\left(2^{j}\right)\right)
$$

concluímos que $K<\infty$.

Parte (iii): Temos que (3.9) é equivalente a

$$
|Q|^{\frac{\mu}{n}-\frac{1}{p}}\left(\int_{Q} \delta_{a} w\right)^{1 / q}\left(\frac{1}{|Q|} \int_{Q}\left(\delta_{a} v\right)^{-r p^{\prime} / p}\right)^{1 / r p^{\prime}} \leq C(a) a^{\gamma-n+1}
$$

Logo, pelo teorema 2.23 temos

$$
\left\|M_{Q_{1}, \mu} f\right\|_{L_{\delta_{a} w}^{q}} \leq c C(a) a^{\gamma-n+1}\|f\|_{L_{\delta_{a} v}^{p}}
$$

e pela equivalência entre (3.25) e (3.26) temos

$$
\left\|\delta_{a}^{-1} M_{Q_{1}, \mu} \delta_{a} f\right\|_{L_{w}^{q}} \leq c C(a) a^{\gamma-n+1}\|f\|_{L_{v}^{p}}
$$

Para $q \geq 1, L_{w}^{q}$ é um espaço de Banach, assim por (3.28) e (3.35),

$$
\begin{aligned}
\left\|M_{S, \mu} f\right\|_{L_{w}^{q}} & \leq C \sum_{k=0}^{\infty} 2^{-k(\gamma-n+1)}\left\|\delta_{2^{-k}} M_{Q_{1}, \mu} \delta_{2^{k}} f\right\|_{L_{w}^{q}} \\
& \leq C \sum_{k=0}^{\infty} 2^{-k(\gamma-n+1)} c C\left(2^{k}\right) 2^{k(\gamma-n+1)}\|f\|_{L_{v}^{p}} \\
& =C c \sum_{k=0}^{\infty} C\left(2^{k}\right)\|f\|_{L_{v}^{p}} \leq B\|f\|_{L_{v}^{p}}
\end{aligned}
$$

com $B \leq c b C$, onde $b$ é a constante em (3.7).

Prova do teorema 3.10: Para provar a afirmação necessária $(i)$, suponhamos que valha (3.11). Seja $\left(R, R^{*}\right) \in \mathcal{B}\left(R_{a}, R_{a}^{*}\right)$. Suponhamos primeiro que $p>1$ e seja $f=v^{-p^{\prime} / p} \chi_{R}$. Se $\xi$ denota o centro de $R, R^{*}$, então para algum $t>0$ temos

$$
R=\xi+t R_{a} \quad \text { e } \quad R^{*}=\xi+t R_{a}^{*}
$$


Se $x \in R^{*}$, podemos facilmente encontrar $y \in t Q_{1}$ tal que $z=x-y$ está em $R$, para que $R$ esteja contido no retângulo $z+2 t R_{a}$. Então como por (3.29) $M_{S, Q_{1}, \mu} f(x) \geq c M_{R_{a}, Q_{1}, \mu} f(x)$ e como $x \in z+t Q_{1} \subseteq z+2 t Q_{1}, R \subseteq z+2 t R_{a}$, temos

$$
M_{S, Q_{1}, \mu} f(x) \geq c(2 t)^{\mu-n}\left(\int_{R} v^{-p^{\prime} / p} d y\right) \chi_{R^{*}}(x) .
$$

Substituindo esta desigualdade em (3.11), usando $t^{n}=|R| /\left|R_{a}\right| \mathrm{e}$

$$
\|f\|_{L_{v}^{p}}=\left(\int_{R} v^{-p^{\prime} / p} d y\right)^{1 / p}
$$

conseguimos (3.12), assumindo $\int_{R} v^{-p^{\prime} / p} d y \neq 0, \infty$. Se $\int_{R} v^{-p^{\prime} / p} d y=0$, não há nada a provar e se $\int_{R} v^{-p^{\prime} / p} d y=\infty$, podemos usar o mesmo argumento "standard" usado no teorema 2.10.

Provemos agora para $p=1$. Seja $A=\operatorname{infess}\{v(x) ; x \in R\}$. Assim, se $\varepsilon>0$ então $E=\{x \in R ; v(x) \leq A+\varepsilon\}$ tem medida positiva. Pondo $f=\chi_{E}$, pelos mesmos argumentos acima, obtemos $M_{S, Q_{1}, \mu} f(x) \geq c(2 t)^{\mu-n}|E| \chi_{R^{*}}(x)$. Também $\|f\|_{L_{v}^{1}} \leq(A+\varepsilon)|E|$. Substituindo este resultado em (3.11), cancelando $|E|$, e fazendo $\varepsilon \rightarrow 0$, temos (3.13).

Para provar (ii) do teorema 3.10, mostraremos que para cada $a \geq 1$, (3.14) implica a estimativa do tipo fraco

$$
w\left(\left\{x ; M_{R_{a}, Q_{1}, \mu} f(x)>s\right\}\right) \leq\left(\frac{D C(a)\|f\|_{L_{v}^{p}}}{s}\right)^{q}
$$

com $D$ uma constante independente de $a$. Disto, a estimativa do tipo fraco para $M_{S, Q_{1}, \mu}$ segue pelo mesmo argumento usado para o operador centrado, que para $q>1$ é

$$
\left\|M_{S, Q_{1}, \mu} f\right\|_{L_{w}^{q, \infty}} \leq C \sum_{k=0}^{\infty}\left\|M_{R_{2^{k}}, Q_{1}, \mu} f\right\|_{L_{w}^{q, \infty}} \leq C^{\prime} \sum_{k=0}^{\infty} C\left(2^{k}\right)\|f\|_{L_{v}^{p}} \leq C^{\prime \prime}\|f\|_{L_{v}^{p}}
$$

onde a primeira desigualdade decorre de (3.30) e do fato de que $L_{w}^{q, \infty}$ é um espaço de Banach para $q>1$ (teorema 1.13), a segunda desigualdade segue de (3.36) e a desigualdade final de (3.7). O caso $q=1$ decorre do lema 3.31, de modo análogo ao caso $q=1$ na prova da parte $($ ii) do teorema 3.1.

Provemos agora que (3.14) implica (3.36). Para $p>1$, seja $f \geq 0, f \in L_{v}^{p}$ e seja $s>0$. Seja $E=\left\{x: M_{R_{a}, Q_{1}, \mu} f(x)>s\right\}$. Para cada $x \in E$ podemos 
encontrar $\xi \in \mathbb{R}^{n}$ e $t>0$ tal que $x \in \xi+t Q_{1}$ e

$$
t^{\mu-n} \int_{R(x)} f(y) d y>s
$$

onde $R(x)=\xi+t R_{a}$. Escrevendo $R^{*}(x)=\xi+t R_{a}^{*}$, como $t^{n}=|R| /\left|R_{a}\right|$, pela desigualdade de Hölder e (3.14) obtemos

$$
\begin{aligned}
s & <\left(\frac{|R|}{\left|R_{a}\right|}\right)^{\frac{\mu}{n}-1} \int_{R(x)} f(y) d y \\
& \leq\left(a^{\gamma-n+1}|R|\right)^{\frac{\mu}{n}-1}\left(\int_{R(x)} f^{p} v d y\right)^{1 / p}\left(\int_{R(x)} v^{-p^{\prime} / p} d y\right)^{1 / p^{\prime}} \\
& \leq C(a)\left(\int_{R(x)} f^{p} v d y\right)^{1 / p}\left(\int_{R^{*}(x)} w d y\right)^{-1 / q}
\end{aligned}
$$

resultando

$$
s^{q}\left(\int_{R^{*}(x)} w d y\right)<C(a)^{q}\left(\int_{R(x)} f^{p} v d y\right)^{q / p} .
$$

Podemos agora aplicar o lema de recobrimento 1.11 para $E$ e da cobertura $\left\{R^{*}(x): x \in E\right\}$ escolher uma subcoleção enumerável $\left\{R_{j}^{*}\right\}$ a qual cobre $E \mathrm{e}$ tem sobreposição limitada. Seja $N$ a constante para a qual $\sum_{x \in R_{j}^{*}} \chi_{R_{j}^{*}}(x) \leq$ $N$, dependendo somente de $n$.

Se os $R_{j}$ são os retângulos correspondentes tal que $\left(R_{j}, R_{j}^{*}\right) \in \mathcal{B}\left(R_{a}, R_{a}^{*}\right)$, então como $q \geq p$, temos

$$
\begin{aligned}
s^{q} w\left(\left\{x: M_{R_{a}, Q_{1}, \mu} f(x)>s\right\}\right) & \leq s^{q} \sum_{j} \int_{R_{j}^{*}} w d y \\
& \leq C(a)^{q} \sum_{j}\left(\int_{R_{j}} f^{p} v d y\right)^{q / p} \\
& \leq C(a)^{q}\left(\sum_{j} \int_{R_{j}} f^{p} v d y\right)^{q / p} \\
& \leq N C(a)^{q}\|f\|_{L_{v}^{p}}^{q}
\end{aligned}
$$

dando o resultado desejado. $\mathrm{O}$ argumento para $p=1$ é similar. 


\subsection{Desigualdades dos tipos forte e fraco para o operador maximal centrado $M_{S, \mu}$, caso $S$ estrelado em relação à origem.}

Nesta seção apresentaremos uma versão do teorema 3.1 para conjuntos estrelados em relação à origem. Para isto precisaremos dos seguintes resuitados:

Definição 3.37 Dado um conjunto estrelado $S$ centrado na origem, diremos que uma coleção de retângulos abertos $\left\{R_{j}\right\}$ com orientação arbitrária é uma cobertura estrelada de $S$ se:

(i) Para cada $j, R_{j}$ contém a origem no seu eixo maior;

(ii) $S \subseteq \cup_{j} R_{j}$;

(iii) $\sum\left|R_{j}\right| \leq c|S|$ para algum $c>0$.

Além disso, se pudermos escolher $\left\{R_{j}\right\}$ tal que para algum $c<1, c R_{j} \subseteq S$ para todo $j$, então diremos que $\left\{R_{j}\right\}$ é uma cobertura estrelada própria de $S$. Finalmente, se cada dos retângulos $R_{j}$ está centrado na origem, então diremos que $\left\{R_{j}\right\}$ é uma cobertura estrelada centrada.

Lema 3.38 (i) Todo conjunto estrelado $S$ tem uma cobertura estrelada $\left\{R_{j}\right\}$ e podemos escolher $\left\{R_{j}\right\}$ sendo uma cobertura estrelada centrada.

(ii) Se $S$ é estrelado e aberto, então ele admite uma cobertura estrelada própria $\left\{R_{j}\right\}$ e podemos escolher $\left\{R_{j}\right\}$ tal que $c R_{j} \subseteq S$ para cada $j$, com $c<1$ dependendo somente de $n$ e $|S| \approx \sum\left|R_{j}\right|$, com constantes de comparabilidade dependendo somente de $n$. Finalmente, se $S$ é também simétrico com relação a origem, então podemos ainda escolher a cobertura estrelada própria tal que esta seja também uma cobertura estrelada centrada.

\section{Prova:}

Para provarmos $(i)$, escrevamos $S=\left\{r \theta ; \theta \in S^{n-1}, 0 \leq r<\rho(\theta)\right\}$ e seja $O_{j}=\left\{\theta \in S^{n-1}: \rho(\theta)>2^{j-1}\right\}, j \in \mathbb{Z}$. Como $\rho$ é semicontínua inferiormente, $O_{j}$ é aberto e podemos assim cobrir $O_{j}$ por $\operatorname{discos} D_{j k}=\{\theta \in$ $\left.S^{n-1}:\left|\theta-\theta_{j k}\right|<\varepsilon_{j k}\right\}$ tais que $\sum_{k}\left|D_{j k}\right| \approx\left|O_{j}\right|$. Para cada disco $D_{j k}$, fixemos um retângulo $R_{j k}$ com eixo maior na direção $\theta_{j k}$ tal que $R_{j k}$ é o menor 
retângulo contendo o "cone" $\left\{r \theta: \theta \in D_{j k},-2^{j}<r<2^{j}\right\}$. Notemos que $R_{j k}$ é único a menos de rotação sobre seu eixo maior, e qualquer escolha serve. Então para cada $j,\left|R_{j k}\right| \approx 2^{n j}\left|D_{j k}\right|$, com constantes de comparabilidade dependendo somente de $n$, resultando:

$$
\sum_{j} \sum_{k}\left|R_{j k}\right| \approx \sum_{j} 2^{n j}\left|O_{j}\right| \approx \frac{1}{n}\|\rho\|_{n}^{n}=c_{n}|S| .
$$

Para completar a prova de $(i)$, observemos que

$$
S \subseteq \cup_{j}\left\{r \theta: \theta \in O_{j}, 0 \leq r<2^{j}\right\} \subseteq \cup_{j} R_{j k} .
$$

Para provar (ii), como $S$ é aberto, segue que $\rho \geq c>0$ e que $\rho$ é semicontínua inferiormente. Por conveniência, suponhamos que $c=1$. Definamos os conjuntos $O_{j}$ como na prova de $(i)$, exceto que desta vez consideraremos somente $j \geq 0$, notando que $O_{j}$ é aberto para $j>0$, e $O_{0}=S^{n-1}$. Com $O_{0}$, associamos o retângulo $R_{01}=(1 / \sqrt{n}) Q_{1}$ e com $O_{j}, j>0$, associamos a coleção de retângulos $R_{j k}$ possivelmente infinita como segue:

Primeiro, escrevamos $O_{j}$ como a união dos discos $D(w, r)=\left\{\theta \in S^{n-1}\right.$ : $|\theta-w|<r\} \operatorname{com} w \in O_{j}$ e $r=2^{-j} \operatorname{dist}\left(w, \partial O_{j}\right)$. Por um processo de seleção "standard" podemos escolher desta coleção, discos disjuntos $D_{j k}=D\left(\theta_{j k}, r_{j k}\right)$ tais que $O_{j} \subseteq \cup_{k} D\left(\theta_{j k}, 5 r_{j k}\right)$. Seja $C_{j k}$ o menor cilindro com eixo paralelo a $\theta_{j k}$ contendo o cone $C=\left\{r \theta: \theta \in D_{j k}, 0 \leq r<2^{j-1}\right\}$ e escolha $\tilde{R}_{j k}$ como sendo um dos maiores retângulos contidos em $C_{j k}$ tal que para qualquer $z \in \tilde{R}_{j k}$, dist $(z, 0) \leq 2^{j-1}$. Então $\tilde{R}_{j k}$ contém a origem na interseção de uma face com o eixo maior.

Afirmamos que $\tilde{R}_{j k} \subset S$. De fato, seja $z \in \tilde{R}_{j k}$. Como $\rho \geq c=1 S$ contém a bola unitária $B_{1}$ centrada na origem. Assim se $z \in \tilde{R}_{j k} \cap B_{1}, z \in S$. Suponhamos então que $z \in \tilde{R}_{j k} \backslash B_{1}$. Observemos que a interseção do cone $C$ com $S^{n-1}$ é um disco $D_{j k}$ com diâmetro que é aproximadamente $2^{-j}$ vezes o diâmetro da base do cone, logo se ampliarmos $D_{j k}$ por um fator de $2^{j}$ obteremos um disco $\tilde{D}_{j k}$ em $S^{n-1}$ que contém a interseção de $\tilde{R}_{j k} \operatorname{com} S^{n-1}$. Tomemos o cone $\tilde{C}=\left\{r \theta ; \theta \in \tilde{D}_{j k}, 0 \leq r \leq 2^{j-1}\right\}$, portanto $R_{j k} \backslash B_{1} \subset \tilde{C}$, ou seja, $z$ é da forma $r \theta, \theta \in \tilde{D}_{j k} \operatorname{com} 1<r \leq 2^{j-1}$. Ainda $\tilde{D}_{j k} \subset O_{j}$ pois construimos os discos $D_{j k}$ tais que possam ser ampliados por $2^{j}$ e ainda estarem contidos em $O_{j}$. Assim, como $\theta \in \tilde{D}_{j k} \subset O_{j}, \rho(\theta)>2^{j-1}$, logo $1<r \leq 2^{j-1}<\rho(\theta)$, ou seja $z \in S$.

Podemos estender $\tilde{R}_{j k}$ um pouco na direção $-\theta_{j k}$ para obtermos um retângulo (novamente denotado por $\tilde{R}_{j k}$ ) que contém própriamente a ori- 
gem e está contido em $S$. Além do mais se $S$ é simétrico com relação a origem podemos de fato estender $\tilde{R}_{j k}$ tal que este esteja centrado na origem.

Observemos que $\left|\tilde{R}_{j k}\right| \approx 2^{n j}\left|D_{j k}\right|$ e que $\left|O_{j}\right| \approx \sum_{k}\left|D_{j k}\right|$. Também, existe uma constante positiva $c_{0}<1$ dependendo somente de $n$ tal que $D\left(\theta_{j k}, c_{0} r_{j k}\right)$ está contido na projeção da base de $\tilde{R}_{j k}$ sobre a esfera unitária. Assim se $C=5 / c_{0}$, então

$$
S \cap\left\{2^{j-1}<|x|<2^{j}\right\} \subseteq \cup_{k} C \tilde{R}_{j k} .
$$

Assim a coleção $\left\{R_{j k}\right\}_{j, k}$ para $R_{j k}=C \tilde{R}_{j k}$ é a cobertura estrelada própria desejada.

Para cada retângulo $R$ contendo a origem, podemos associar uma transformação linear $\delta_{R}$ de determinante positivo tal que $R=\delta_{R} Q_{R}$, onde $Q_{R}$ é um cubo unitário contendo a origem, com arestas paralelas aos lados de $R$ e $\delta_{R}$ é diagonal em relação a base ortonormal de vetores unitários paralelos aos lados de $R$, e que a entrada diagonal correspondente a um lado de $R$ é o comprimento de $R$ naquela direção. Então $\operatorname{det} \delta_{R}=|R|$.

O teorema seguinte é análogo ao teorema 3.1.

Teorema 3.39 Sejam $1 \leq p \leq q<\infty$ e $0 \leq \mu<n$ e sejam $v, w$ pesos.

(i) Seja $S$ um conjunto aberto estrelado e simétrico com relação à origem e suponhamos que $M_{S, \mu}$ satisfaça a estimativa do tipo fraco

$$
w\left(\left\{x ; M_{S, \mu} f(x)>s\right\}\right) \leq\left(\frac{B\|f\|_{L_{v}^{p}}}{s}\right)^{q} .
$$

Então, para cada retângulo $R$ contido em $S$ e contendo a origem num eixo e para todo cubo $Q$, os pesos $v$ e w satisfazem

$$
\begin{gathered}
|Q|^{\frac{\mu}{n}-1}\left(\int_{Q} \delta_{R} w\right)^{1 / q}\left(\int_{Q}\left(\delta_{R} v\right)^{-p^{\prime} / p}\right)^{1 / p^{\prime}} \leq \frac{C B}{|R|}, \quad p>1, \\
|Q|^{\frac{\mu}{n}-1}\left(\int_{Q} \delta_{R} w\right)^{1 / q} \operatorname{supess}_{Q}\left(\delta_{R} v\right)^{-1} \leq \frac{C B}{|R|}, \quad p=1,
\end{gathered}
$$

onde $C$ é uma constante independente de $v, w, e R$.

(ii) Reciprocamente, suponhamos que $S$ seja um conjunto estrelado, $\left\{R_{j}\right\}$ seja uma cobertura estrelada, e sejam $C_{j}$ constantes tais que para todo 
cubo $Q$,

$$
|Q|^{\frac{\mu}{n}-1}\left(\int_{Q} \delta_{R_{j}} w\right)^{1 / q}\left(\int_{Q}\left(\delta_{R_{j}} v\right)^{-p^{\prime} / p}\right)^{1 / p^{\prime}} \leq \frac{C_{j}}{\left|R_{j}\right|}, \quad p>1,
$$

$e$

$$
|Q|^{\frac{\mu}{n}-1}\left(\int_{Q} \delta_{R_{j}} w\right)^{1 / \tilde{q}} \operatorname{supess}_{Q}\left(\delta_{R_{j}} v\right)^{-1} \leq \frac{\bar{C}_{j}}{\left|R_{j}\right|}, \quad p=1 .
$$

Se, também

$$
\sum_{j} C_{j}=b<\infty, \quad q>1
$$

$e$

$$
\sum_{j} C_{j}\left(1+\log ^{+} \frac{1}{C_{j}}\right)=b<\infty, \quad q=p=1 .
$$

Então, $M_{S, \mu}$ satisfaz a estimativa do tipo fraco 3.40 , com $B \leq c b$ para alguma constante $c$ independente de $v, w$.

(iii) Sejam $S$ e $R_{j}$ como em (ii). Se $1<p \leq q<\infty$ e $v$, w satisfazem para algum $r>1$,

$$
|Q|^{\frac{\mu}{n}-\frac{1}{p}}\left(\int_{Q} \delta_{R_{j}} w\right)^{1 / q}\left(\frac{1}{|Q|} \int_{Q}\left(\delta_{R_{j}} v\right)^{-r p^{\prime} / p}\right)^{1 /\left(r p^{\prime}\right)} \leq \frac{C_{j}}{\left|R_{j}\right|}
$$

para todo $j$ e todo cubo $Q$, com $\left\{C_{j}\right\}$ satisfazendo (3.45) e (3.46), então $M_{S, \mu}$ satisfaz a estimativa do tipo forte

$$
\left\|M_{S, \mu} f\right\|_{L_{w}^{q}} \leq B\|f\|_{L_{v}^{p}}
$$

com $B \leq c b$ e c independente de $w, v$ e $f$.

\section{Prova:}

Do lema 3.38, vemos que se $S$ é estrelado com relação a origem e $\left\{R_{j}\right\}$ é uma cobertura estrelada como garantida pelo lema, então

$$
\chi_{S}(x) \leq \sum_{j=0}^{\infty} \chi_{R_{j}}(x)
$$


com as integrais dos dois lados sendo comparáveis.

Ainda, o lema 3.38 mostra que se $S$ é aberto, podemos escolher a cobertura satisfazendo

$$
\chi_{S}(x) \geq \chi_{R_{j}}(x / c)
$$

onde $c$ é a constante tal quie $c P_{j} \subseteq S$ para todo $j$.

Na parte $(i)$ do teorema, como $S$ é simétrico com relação à origem além de aberto, pelo lema $3.38, S$ admite uma cobertura estrelada própria por retângulos centrados na origem. Portanto, se $R$ é um retângulo centrado na origem e se $\delta_{R}, Q_{R}$ são respectivamente a correspondente transformação linear e o cubo unitário tal que $R=\delta_{R} Q_{R}$ como mencionado anteriormente, então $Q_{R}$ está também centrado na origem e, portanto, é comparável a $Q_{1}$ e à bola unitária, isto é, existem constantes positivas $c_{1}$ e $c_{2}$ tais que $c_{1} Q_{R} \subseteq$ $Q_{1} \subseteq c_{2} Q_{R}$ onde $c_{1}$ e $c_{2}$ não dependem de $R$.

Nas partes (ii) e (iii) do teorema, apesar de $S$ não ser necessariamente aberto e nem simétrico em relação à origem, é suficiente cobrir $S$ por retângulos contendo a origem, pois se $R$ é um tal retângulo com $\delta_{R}, Q_{R}$ associados, então $Q_{R}$ contém a origem e assim $Q_{R} \subseteq c Q_{1}$ com $c$ uma constante dependendo somente de $n$. A comparação contrária falha, desde que a origem possa estar arbitráriamente próxima ao fecho da fronteira de $Q_{R}$.

Assim, em ambos os casos é possível relacionar operadores envolvendo médias sobre $Q_{R}$ por correspodentes operadores envolvendo médias sobre $Q_{1}$. Uma afirmação similar vale com $Q_{1}$ trocado pela bola unitária centrada na origem.

Com isto, observemos que o teorema 3.39 segue quase como o teorema 3.1, pois quando (3.47) vale, então por (3.21) (lembrando que $\left|\operatorname{det} \delta_{R}\right|=|R|$, e pondo $Q_{j}=Q_{R}$ para $R=R_{j}$ ) temos

$$
\begin{aligned}
M_{S, \mu} f(x) & \leq c \sum_{j=0}^{\infty} M_{R_{j}, \mu} f(x)=c \sum_{j=0}^{\infty}\left|R_{j}\right| \delta_{R_{j}}^{-1} M_{Q_{j}, \mu} \delta_{R_{j}} f(x) \\
& \leq c^{\prime} \sum_{j=0}^{\infty}\left|R_{j}\right| \delta_{R_{j}}^{-1} M_{Q_{1}, \mu} \delta_{R_{j}} f(x) .
\end{aligned}
$$

A última desigualdade de (3.49) segue pela troca de $Q_{R}$ com o múltiplo fixo de $Q_{1}$ ou a bola unitária que contém $Q_{R}$ para todo $j$. 
Reciprocamente, se $R$ é um retângulo centrado na origem contido em $S$, então por (3.48)

$$
\begin{aligned}
M_{S, \mu} f(x) & \geq c M_{R, \mu} f(x)=c|R| \delta_{R}^{-1} M_{Q_{R}, \mu} \delta_{R} f(x) \\
& \geq c|R| \delta_{R}^{-1} M_{Q_{1}, \mu} \delta_{R} f(x),
\end{aligned}
$$

oñde a última desigualdade usa o fato que $Q_{1} \in Q_{R}$ são comparááveis se $R$ está centrado na origem, com constantes de comparabilidade dependendo somente de $n$.

Arguindo como no teorema 3.1 , o teorema 3.39 para $M_{S, \mu}$ segue então de (3.49) e (3.50), usando a equivalência de (3.23) e (3.24), e de (3.25) e (3.26).

Nos artigos estudados, não temos o que seria o análogo ao teorema 3.10 para conjuntos estrelados $S$ mais gerais, ou seja, um resultado do tipo fraco sobre o operador maximal não centrado $M_{S, Q_{1}, \mu}$ para conjuntos $S$ mais gerais que $S=S_{\rho}$ para $\rho(\theta)=\left|\theta_{n}\right|^{-1 /(\gamma+1)}$, contudo faremos um estudo deste caso na seção 3.4 .

\subsection{Desigualdade do tipo forte para o opera- dor maximal não centrado $M_{S, E, \mu}$.}

Nesta seção provaremos o teorema 0.10 da introdução, o qual enuciamos novamente abaixo:

Sejam $\gamma>0$ e $S=S_{\gamma}$. Sejam $0 \leq \mu<n, 1<p \leq q<\infty$ e suponhamos que exista $r>1$ tal que

$$
|R|^{\frac{\mu}{n}-\frac{1}{p}} w\left(R^{*}\right)^{\frac{1}{q}}\left(\frac{1}{|R|} \int_{R} v^{\left(-p^{\prime} r\right) / p} d x\right)^{\frac{1}{r p^{\prime}}} \leq C(a)\left|R_{a}\right|^{\frac{\mu}{n}-1}
$$

para todo $R \in \mathcal{B}\left(R_{a}\right)$ e todo $a \geq 1$, onde $C(a)$ é uma função não crescente que satisfaça

$$
\int_{1}^{\infty} C(a) \frac{d a}{a}=b
$$

Então,

$$
\left\|M_{S_{\gamma}, Q_{1}, \mu} f\right\|_{L_{w}^{q}} \leq c\|f\|_{L_{v}^{p} .}
$$

Para esta prova precisaremos de vários resultados: seja $\mathcal{B}$ a família de todas as translações e dilatações de um retângulo fixado $R_{\mathcal{B}}$ (isto é, $\mathcal{B}=$ 
$\mathcal{B}\left(R_{\mathcal{B}}\right)$ na nossa notação). Claro que $R_{\mathcal{B}}$ não é unicamente determinado por $\mathcal{B}$, mas suas excentricidades (razão dos lados) são, e podemos assumir sem perda de generalidade que seu primeiro comprimento é 1 . Assim, por exemplo, podemos tomar o retângulo básico em $\mathcal{B}\left(R_{a}\right)$ como tendo lados de comprimentos $1, . ., 1, a^{-\gamma-1}$. Para cada $R \in \mathcal{B}$ associamos um conjunto (não necessariamente um retângulo) $R^{*}$ tal que o seguinte vale:

$$
\text { Se } R_{1}, R_{2} \in \mathcal{B} \text { e } R_{1} \subset R_{2} \text { então } R_{1}^{*} \subset R_{2}^{*} \text {. }
$$

Por exemplo, o par $\left(R, R^{*}\right)$ dos transladados e dilatados de $\left(R_{a}, R_{a}^{*}\right)$ definido anteriormente tem esta propriedade. Mais geralmente, se $R_{\mathcal{B}}^{*}$ é definido como sendo um retângulo qualquer contendo $R_{\mathcal{B}}$ e dado $R \in \mathcal{B}, R=z+t R_{\mathcal{B}}$, definamos $R^{*}=z+t R_{\mathcal{B}}^{*}$, então o par $\left(R, R^{*}\right) \operatorname{satisfaz~(3.51.)~}$

Para uma tal coleção de pares e $0 \leq \alpha<1$, definamos:

$$
M_{\alpha} f(x)=\sup _{\substack{R \in \mathcal{B} \\ x \in R^{*}}}|R|^{\alpha-1} \int_{R}|f(y)| d y .
$$

É claro que este operador depende de $\mathcal{B}$ e da escolha dos conjuntos $R^{*}$, embora para simplificar nossa notação, não citaremos esta dependência. Seja $\mathcal{B}=\mathcal{B}\left(R_{\mathcal{B}}\right)$ uma família de retângulos como descrito acima, com conjuntos associados $R^{*}$ que satisfazem (3.51). Sejam $e_{1}, \ldots, e_{n}\left(e_{1}=1\right.$, digamos) os comprimentos dos lados de $R_{\mathcal{B}}$ e seja $\mathcal{B}^{d y}$ a correspondente rede de retângulos diádicos da forma

$$
\left[\frac{m_{1} e_{1}}{2^{j}}, \frac{\left(m_{1}+1\right) e_{1}}{2^{j}}\right] \times \ldots . . \times\left[\frac{m_{n} e_{n}}{2^{j}}, \frac{\left(m_{n}+1\right) e_{n}}{2^{j}}\right]
$$

para $j, m_{1}, \ldots, m_{n}=0, \pm 1, \pm 2, \ldots$ Cada retângulo de $\mathcal{B}^{d y}$ está também em $\mathcal{B}$. Definamos

$$
M_{\alpha}^{d y} f(x)=\sup _{\substack{R \in \mathcal{B}^{d y} \\ x \in R^{*}}}|R|^{\alpha-1} \int_{R}|f(y)| d y,
$$

e para $z \in \mathbb{R}^{n}$, definamos

$$
M_{\alpha}^{d y, z} f(x)=\sup _{\substack{R \in \mathcal{B}^{d y} \\ x \in(R+z)^{*}}}|R+z|^{\alpha-1} \int_{R+z}|f(y)| d y .
$$

É claro que $|R+z|=|R|$. 
Lema 3.53 Se $1 \leq q \leq \infty$ e w é um peso, então

$$
\left\|M_{\alpha} f\right\|_{L_{w}^{q}} \leq c \sup _{z \in \mathbb{R}^{n}}\left\|M_{\alpha}^{d y, z} f\right\|_{L_{w}^{q}}
$$

com c dependendo de a e n, mas não de $\mathcal{B}$ ou $f$.

Prova: Fixemos $R \in \hat{\mathcal{B}}$ e consideremos a coleção daqueles $R_{1} \in \mathfrak{B}^{d y}$ cujos comprimentos dos lados são duas vezes os comprimentos dos lados de $R$, respectivamente, e consideremos $\mathbb{R}^{n}$ como a união de tais $R_{1}$. Claro que $\left|R_{1}\right| \approx|R|$ para cada $R_{1}$ com constante de equivalência dependendo somente de $n$. Um simples argumento usando translações mostra que

$$
\left|\left\{z \in \mathbb{R}^{n}: R \subset R_{1}+z\right\}\right| \geq c\left|R_{1}\right|
$$

com $c>0$ dependendo somente de $n$. Também, com $R$ ainda fixo, os conjuntos $\left\{z \in \mathbb{R}^{n}: R \subset R_{1}+z\right\}$ são essencialmente disjuntos para diferentes (não sobrepostos) $R_{1}$. Seja

$$
E\left(R_{1}\right)=\left\{z \in \mathbb{R}^{n}: R \subset R_{1}+z, R^{*} \subset\left(R_{1}+z\right)^{*}\right\}
$$

e notemos por (3.51) que $E\left(R_{1}\right)$ é o mesmo que o conjunto $\left\{z \in \mathbb{R}^{n}: R \subset\right.$ $\left.R_{1}+z\right\}$ acima. Também se $x \in R^{*}$ e $z \in E\left(R_{1}\right)$, então

$$
|R|^{\alpha-1} \int_{R}|f(y)| d y \leq c\left|R_{1}+z\right|^{\alpha-1} \int_{R_{1}+z}|f(y)| d y \leq c M_{\alpha}^{d y, z} f(x),
$$

pois $\left|R_{1}+z\right|=\left|R_{1}\right| \approx|R|, R \subset R_{1}+z$ e $x \in\left(R_{1}+z\right)^{*}$. A constante $c$ depende somente de $n, \alpha$.

Seja $\Omega=\cup_{R_{1}} E\left(R_{1}\right)$. Observemos que a desigualdade (3.54) vale se $x \in R^{*}$ e $z \in \Omega$. Observemos também que $\left|E\left(R_{1}\right)\right| \geq c\left|R_{1}\right|$ para cada $R_{1}$ e que os $E\left(R_{1}\right)$ são essencialmente disjuntos para distintos $R_{1}$.

Agora, tomando o $\sup _{\substack{R \in \mathcal{B} \\ x \in R^{*}}}$ em (3.54) temos

$$
M_{\alpha} f(x) \leq c M_{\alpha}^{d y, z} f(x) .
$$

Seja $R^{\prime}=\cup_{j=1}^{k} E\left(R_{1}^{j}\right), \operatorname{logo}$

$$
\left|R^{\prime}\right| M_{\alpha} f(x) \leq c \int_{\cup_{j=1}^{k} E\left(R_{1}^{j}\right)} M_{\alpha}^{d y, z} f(x) d z .
$$


Assim,

$$
\begin{aligned}
\left\|M_{\alpha} f\right\|_{L_{w}^{q}} & \leq \frac{c}{\left|R^{\prime}\right|}\left\|\int_{\cup_{j=1}^{k} E\left(R_{1}^{j}\right)} M_{\alpha}^{d y, z} f d z\right\|_{L_{w}^{q}} \\
& \leq \frac{c}{\left|R^{\prime}\right|} \int_{\bigcup_{j=1}^{k} E\left(R_{1}^{j}\right)}\left\|M_{\alpha}^{d y, z} f\right\|_{L_{w}^{q}} d z, \quad \text { por Minkowski, } \\
& =\frac{c}{\left|R^{\prime}\right|} \sum_{j=1}^{k} \int_{E\left(R_{1}^{j}\right)}\left\|M_{\alpha}^{d y, z} f\right\|_{L_{w}^{q}} d z, \quad \text { pois os } E\left(R_{1}^{j}\right) \text { são disjuntos, } \\
& \leq c \sup _{\mathbb{R}^{n}}\left\|M_{\alpha}^{d y, z} f\right\|_{L_{w}^{q}} \frac{\sum_{j=1}^{k}\left|E\left(R_{1}^{j}\right)\right|}{\left|R^{\prime}\right|} \\
& \leq c \sup _{z \in \mathbb{R}^{n}}\left\|M_{\alpha}^{d y, z} f\right\|_{L_{w}^{q}}, \quad \text { pois }\left|R^{\prime}\right|=\sum_{j=1}^{k}\left|E\left(R_{1}^{j}\right)\right| .
\end{aligned}
$$

O seguinte teorema será de grande importância para a demonstração do teorema 0.10 .

Teorema 3.55 Sejam $1<p \leq q<\infty$ e $0 \leq \alpha<1$ e seja $M_{\alpha}$ definido por (3.52). Se existe $r>1$ tal que

$$
|R|^{\alpha-\frac{1}{p}} w\left(R^{*}\right)^{1 / q}\left(\frac{1}{|R|} \int_{R} v^{\left(-p^{\prime} r\right) / p} d x\right)^{1 / r p^{\prime}} \leq C,
$$

para todo $R \in \mathcal{B}$, então

$$
\left\|M_{\alpha} f\right\|_{L_{w}^{q}} \leq C\|f\|_{L_{v}^{p}}
$$

com uma constante $C$ que é um múltiplo da constante em (3.56) dependendo de $\alpha, n, p$ e q, mas não de $\mathcal{B}$ ou $f$.

\section{Prova:}

Para provar o teorema 3.55 é suficiente pelo lema 3.53 provar o análogo de (3.57) para cada $M_{\alpha}^{d y, z}$, com uma constante independente de $z$. Se trocarmos $f$ por $f v^{-p^{\prime} / p}$, isto significa o seguinte:

$$
\left\|M_{\alpha}^{d y, z}\left(f v^{-p^{\prime} / p}\right)\right\|_{L_{w}^{q}} \leq c\|f\|_{L_{v}^{p}-p^{\prime} / p}
$$


com $c$ igual a um múltiplo da constante em (3.56) dependendo somente de $\alpha, n, p$ e $q$.

Para provar (3.58), fixemos $z$ e $f \geq 0$ e para $k=0, \pm 1, \pm 2, \ldots$, seja

$$
\Omega_{k}=\left\{x \in \mathbb{R}^{n}: M_{\alpha}^{d y, z}\left(f v^{-p^{\prime} / p}\right)(x)>2^{k n}\right\} .
$$

Então, $x \in \Omega_{\bar{k}}$ se c somente se existe $R \in \mathcal{B}^{d y}$ tal que $x \in(R+z)^{*}$ e

$$
|R|^{\alpha-1} \int_{R+z} f v^{-p^{\prime} / p} d y>2^{k n}
$$

Em particular, se $R \in \mathcal{B}^{d y}$ e (3.59) vale, então $(R+z)^{*} \subset \Omega_{k}$. Sejam $\left\{R_{j}^{k}\right\}_{j}$ os retângulos diádicos maximais (com respeito a inclusão) em $\mathcal{B}^{d y}$ que satisfazem (3.59). A existência destes retângulos é garantida se $f$ tiver suporte compacto, o que podemos assumir sem perda de generalidade. Pela maximalidade, os $\left\{R_{j}^{k}+z\right\}_{j}$ são não sobrepostos para cada $k$. Ainda mais, se $\bar{R}_{j}^{k}$ é o próximo retângulo diádico contendo $R_{j}^{k}$, então

$$
\left|\bar{R}_{j}^{k}\right|^{\alpha-1} \int_{\bar{R}_{j}^{k}+z} f v^{-p^{\prime} / p} d y \leq 2^{k n}
$$

pela maximalidade e como $\left|\bar{R}_{j}^{k}\right|=2^{n}\left|R_{j}^{k}\right|$, temos

$$
2^{k n}<\left|R_{j}^{k}\right|^{\alpha-1} \int_{R_{j}^{k}+z} f v^{-p^{\prime} / p} d y \leq 2^{n(1-\alpha)} 2^{k n} .
$$

Afirmamos que

$$
\Omega_{k}=\cup_{j}\left(R_{j}^{k}+z\right)^{*} .
$$

Já observemos que cada $\left(R_{j}^{k}+z\right)^{*}$ está em $\Omega_{k}$. Por outro lado, se $x \in \Omega_{k}$ existe um diádico $R \operatorname{com} x \in(R+z)^{*}$ tal que (3.59) vale. Assim $R \subset R_{j_{0}}^{k}$ para algum $j_{o}$ (sendo $R$ maximal ou não) e conseqüentemente $(R+z)^{*} \subset\left(R_{j_{o}}^{k}+z\right)^{*}$ por (3.51). Logo, $x \in\left(R_{j_{o}}^{k}+z\right)^{*}$ e a afirmação segue. Por (3.61),

$$
\Omega_{k} \backslash \Omega_{k+1}=\cup_{j}\left[\left(R_{j}^{k}+z\right)^{*} \backslash \Omega_{k+1}\right]=\cup_{j} E_{j}^{k},
$$

onde

$$
E_{j}^{k}=\left(R_{j}^{k}+z\right)^{*} \backslash \Omega_{k+1}
$$


Assim,

$$
\begin{aligned}
\left\|M_{\alpha}^{d y, z}\left(f v^{-p^{\prime} / p}\right)\right\|_{L_{w}^{q}}^{q} & =\int_{\mathbb{R}^{n}}\left[M_{\alpha}^{d y, z}\left(f v^{-p^{\prime} / p}\right)(x)\right]^{q} w d x \\
& =\sum_{k} \int_{\Omega_{k} \backslash \Omega_{k+1}}\left[M_{\alpha}^{d y, z}\left(f v^{-p^{\prime} / p}\right)(x)\right]^{q} w d x \\
& \leq \sum_{k, j} 2^{(k+1) n q} w\left(E_{j}^{k}\right) \\
& \leq 2^{n q} \sum_{k, j}\left(\left|R_{j}^{k}\right|^{\alpha-1} \int_{R_{j}^{k}+z} f v^{-p^{\prime} / p} d y\right)^{q} w\left(E_{j}^{k}\right) \\
& =2^{n q} \sum_{k, j} w\left(E_{j}^{k}\right)\left[\left|R_{j}^{k}\right|^{\alpha-1} A\left(R_{j}^{k}+z\right)\right]^{q} \cdot \\
& \cdot\left(\frac{1}{A\left(R_{j}^{k}+z\right)} \int_{R_{j}^{k}+z} f v^{-p^{\prime} / p} d y\right)^{q},
\end{aligned}
$$

onde para um retângulo $R$,

$$
A(R)=|R|^{1 / r^{\prime}}\left(\int_{R} v^{-p^{\prime} r / p} d y\right)^{1 / r} .
$$

Estimamos a última soma usando a hipótese (3.56) para os retângulos $R_{j}^{k}+z$ e o fato que $E_{j}^{k} \subset\left(R_{j}^{k}+z\right)^{*}$, obtendo

$$
\begin{aligned}
\left\|M_{\alpha}^{d y, z}\left(f v^{-p^{\prime} / p}\right)\right\|_{L_{w}^{q}}^{q} \leq 2^{n q} C^{q} & \sum_{k, j} A\left(R_{j}^{k}+z\right)^{q / p} \\
& \cdot\left(\frac{1}{A\left(R_{j}^{k}+z\right)} \int_{R_{j}^{k}+z} f v^{-p^{\prime} / p} d y\right)^{q},
\end{aligned}
$$

onde $C$ é a constante em (3.56)

O restante da prova é baseado na aplicação do próximo lema, extraído de [SW], para estimar a soma em (3.62).

Lema 3.63 Seja $\left\{R_{i}\right\}_{i \in I}$ uma coleção de retângulos de uma rede diádica fixada, como por exemplo da forma $\mathcal{B}^{d y}+z$ para $z$ fixado, seja $\beta \geq 1$, e sejam $\left\{a_{i}\right\}_{i \in I}$ números positivos que satisfaçam

(i) $\int_{R_{i}} v^{-p^{\prime} / p} d y \leq c_{o} a_{i}$ 
(ii) $\sum_{j: R_{j} \subset R_{i}} a_{j}^{\beta} \leq c_{o} a_{i}^{\beta}$

para cada $i$, com $c_{o}$ independente de $i$. Então se $1<p<\infty$ e $q=\beta p$,

$$
\left[\sum_{i \in I} a_{i}^{\beta}\left(\frac{1}{a_{i}} \int_{R_{i}}|f| v^{-p^{\prime} / p} d y\right)^{q}\right]^{1 / q} \leq c\|f\|_{L_{v-p^{\prime} / p}^{p}},
$$

com $c$ dependendo de $c_{o}, p$ e $q$, mas não de $f$ ou da rede.

Prova: Consideremos a transformação $T$ definida por:

$$
T(f)=\left(\frac{1}{a_{i}} \int_{R_{i}} f v^{-p^{\prime} / p} d y\right)_{i \in I} .
$$

Afirmamos que $T$ leva $L^{\infty}\left(\mathbb{R}^{n}, v^{-p^{\prime} / p}\right)$ em $\ell^{\infty}\left(I, a_{i}^{\beta}\right)$. De fato, pela condição (i) temos,

$$
\left|\frac{1}{a_{i}} \int_{R_{i}} f v^{-p^{\prime} / p} d y\right| \leq\|f\|_{L_{v^{-p^{\prime} / p}}^{\infty}}\left|\frac{1}{a_{i}} \int_{R_{i}} v^{-p^{\prime} / p} d y\right| \leq\|f\|_{L_{v^{-p^{\prime}} / p}^{\infty}} c_{0}=C^{\prime},
$$

desde que $f \in L^{\infty}\left(\mathbb{R}^{n}, v^{-p^{\prime} / p}\right)$. Seja $b_{i}=\frac{1}{a_{i}} \int_{R_{i}} f v^{-p^{\prime} / p} d y$, portanto pela desigualdade acima temos que $\left|b_{i}\right| \leq C^{\prime}$, para $\forall i \in I$. Logo, dado $\varepsilon>0$, o conjunto $J_{\varepsilon}=\left\{i \in I,\left|b_{i}\right|>C^{\prime}+\varepsilon\right\}$ é vazio e $a\left(J_{\varepsilon}\right)=0$, onde $a(J)=\sum_{i \in J} a_{i}^{\beta}$,

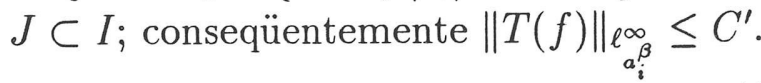

Temos também que $T$ leva $L^{1}\left(\mathbb{R}^{n}, v^{-p^{\prime} / p}\right)$ em $\ell^{\beta}\left(I, a_{i}^{\beta}\right)$ fraco, pela condição (ii), como mostraremos agora.

Para $f$ limitada com suporte compacto em $\mathbb{R}^{n}$ e $\lambda>0$, sejam $\left\{R_{j}\right\}_{j \in J}$ os retângulos maximais diádicos da coleção $\left\{R_{i}\right\}_{i \in I}$ tal que $\frac{1}{a_{i}} \int_{R_{i}}|f| v^{-p^{\prime} / p} d y$ excede $\lambda$ (podemos assumir que a coleção $\left\{R_{j}\right\}_{j \in J}$ é finita). Entâo,

$$
\begin{aligned}
\sum_{i:\left|\frac{1}{a_{i}} \int_{R_{i}} f v^{-p^{\prime} / p} d y\right|>\lambda} a_{i}^{\beta} & \leq \sum_{j \in J} \sum_{i: R_{i} \subset R_{j}} a_{i}^{\beta} \leq \sum_{j \in J} c_{0} a_{j}^{\beta} \text { por }(i i) \\
& \leq \frac{c_{0}}{\lambda^{\beta}} \sum_{j \in J}\left(\int_{R_{j}}|f| v^{-p^{\prime} / p} d y\right)^{\beta} \leq \frac{C}{\lambda^{\beta}}\left(\int_{\mathbb{R}^{n}}|f| v^{-p^{\prime} / p} d y\right)^{\beta}
\end{aligned}
$$

desde que $\beta \geq 1$ e os retângulos maximais $R_{j}, j \in J$, são dois a dois disjuntos. $\mathrm{O}$ teorema de interpolação de Marcinkiewicz completa a prova do lema. 
Se aplicarmos o lema 3.63 para a soma em (3.62) e notarmos que $\int_{R} v^{-p^{\prime} / p}$ $\leq|R|^{1 / r^{\prime}}\left(\int_{R} v^{-p^{\prime} r / p} d y\right)^{1 / r}=A(R)$ pela desigualdade de Hölder, imediatamente obteremos (3.58) de (3.62) se verificarmos

$$
\sum_{k, j: R_{j}^{k} \subset R_{\stackrel{?}{m}}^{m}} A\left(R_{j}^{k}+z\right)^{q / p} \leq c A\left(R_{\ell}^{m}+z\right)^{q / p}
$$

para cada $R_{\ell}^{m}$ e $0<p \leq q<\infty$, com $c$ independente de $\ell, m$ e $z$. Usando a simples desigualdade $\sum a_{i}^{q / p} \leq\left(\sum a_{i}\right)^{q / p}, q \geq p, a_{i} \geq 0$, podemos provar apenas o caso $q=p$. Se $R_{j}^{k}$ é um subconjunto próprio de $R_{\ell}^{m}$, então

$$
2^{m n}<\left|R_{\ell}^{m}\right|^{\alpha-1} \int_{R_{\ell}^{m}+z} f v^{-p^{\prime} / p} d y \leq 2^{k n},
$$

onde a segunda desigualdade segue da maximalidade de $R_{j}^{k}$. Portanto, devemos ter $k>m$ em (3.64) e podemos reescrever o lado esquerdo de (3.64) $(\operatorname{com} q / p=1)$, como

$$
\sum_{k=m}^{\infty} \sum_{j: R_{j}^{k} \subset R_{\ell}^{m}} A\left(R_{j}^{k}+z\right) .
$$

Pela desigualdade de Hölder e da definição de $A(R)$, a soma interna em (3.65) é majorada por

$$
\left(\sum_{j: R_{j}^{k} \subset R_{\ell}^{m}}\left|R_{j}^{k}\right|\right)^{1 / r^{\prime}}\left(\sum_{j: R_{j}^{k} \subset R_{\ell}^{m}} \int_{R_{j}^{k}+z} v^{-p^{\prime} r / p} d y\right)^{1 / r} .
$$

Logo, como $\left\{R_{j}^{k}+z\right\}_{j}$ são não sobrepostos para cada $z$ fixado, o segundo fator é majorado por $\left(\int_{R_{\ell}^{m}+z} v^{-p^{\prime} r / p} d y\right)^{1 / r}$. Também para o primeiro fator, pela primeira desigualdade de (3.60),

$$
\sum_{j: R_{j}^{k} \subset R_{\ell}^{m}}\left|R_{j}^{k}\right| \leq \sum_{j: R_{j}^{k} \subset R_{\ell}^{m}}\left(2^{-k n} \int_{R_{j}^{k}+z} f v^{-p^{\prime} / p} d y\right)^{\frac{1}{1-\alpha}}
$$




$$
\begin{aligned}
& \leq\left(\sum_{j: R_{j}^{k} \subset R_{\ell}^{m}} 2^{-k n} \int_{R_{j}^{k}+z} f v^{-p^{\prime} / p} d y\right)^{\frac{1}{1-\alpha}} \text { pois } \frac{1}{1-\alpha} \geq 1 \\
& \leq\left(2^{-k n} \int_{R_{\ell}^{m}+z} f v^{-p^{\prime} / p} d y\right)^{\frac{1}{1-\alpha}} \\
& \leq\left(2^{-k n}\left|R_{\ell}^{m}\right|^{1-\alpha} 2^{n(1-\alpha)} 2^{m n}\right)^{\frac{1}{1-\alpha}} \quad \text { pela } 2^{a} \text { desigualdade de }(3.60) \\
& =2^{n} 2^{(m-k) n /(1-\alpha)}\left|R_{\ell}^{m}\right| .
\end{aligned}
$$

Combinando estas estimativas, vemos que (3.65) é majorada por

$$
\sum_{k=m}^{\infty}\left(2^{n} 2^{(m-k) n / 1-\alpha)}\left|R_{\ell}^{m}\right|\right)^{1 / r^{\prime}}\left(\int_{R_{\ell}^{m}+z} v^{-p^{\prime} r / p} d y\right)^{1 / r}=c A\left(R_{\ell}^{m}+z\right),
$$

como desejado. Isto prova (3.64) e também completa a prova do teorema (3.55).

Mostraremos agora como o teorema (3.55) implica o teorema 0.10. De fato, se $S$ é um conjunto estrelado em relação à origem, então pelo lema 3.38 , $S$ admite uma cobertura estrelada $\left\{R_{j}\right\}$.

Dado um conjunto estrelado $S$ com relação à origem, fixemos uma cobertura $\left\{R_{j}\right\}$ como acima. Dado um conjunto limitado $E$, definamos $R_{j}^{*}$ como sendo um retângulo contendo ambos $E$ e $R_{j}$. Também, para cada $j$, seja $\mathcal{B}\left(R_{j}\right)$ a coleção de todos os dilatados e transladados de $R_{j}$ e, se $R \in \mathcal{B}\left(R_{j}\right)$, digamos $R=z+t R_{j}$, definamos $R^{*}=z+t R_{j}^{*}$. Os pares $\left(R, R^{*}\right), R \in \mathcal{B}\left(R_{j}\right)$, satisfazem então (3.51).

Teorema 3.66 Sejam $0 \leq \mu<n, 1<p \leq q<\infty, S$ um conjunto estrelado com relação à origem e E um conjunto limitado. Com a notação acima, assumamos que exista $r>1$ tal que

$$
|R|^{\frac{\mu}{n}-\frac{1}{p}} w\left(R^{*}\right)^{1 / q}\left(\frac{1}{|R|} \int_{R} v^{\frac{-r}{p-1}} d x\right)^{1 / r p^{\prime}} \leq C_{R_{j}}\left|R_{j}\right|^{\frac{\mu}{n}-1}
$$

para todo $R \in \mathcal{B}\left(R_{j}\right)$ e que $\sum C_{R_{j}}<\infty$. Então,

$$
\left\|M_{S, E, \mu} f\right\|_{L_{w}^{q}} \leq c\|f\|_{L_{v}^{p}} .
$$


Antes de iniciarmos a prova, observemos que o teorema (3.66) inclui o teorema 0.10 pondo $S=S_{\gamma}, E=Q_{1}$ e $R_{j}=\delta_{a} Q_{1}$ para $a=2^{j}, j=1,2, \ldots$, isto é, $R_{2 j}$ na notação da introdução.

Lembremos que na introdução $R_{a}^{*}$ é o menor retângulo contendo ambos $R_{a}$ e $Q_{1}$. A hipótese adicional no teorema (3.66) de que $\sum C_{R_{j}}<\infty$ equivale então a (3.7). Finalmente, provaremos o teorema 1, para isto escrevemos

$$
\begin{aligned}
M_{S, E, \mu} f(x) & =\sup _{\substack{z \in \mathbb{R}^{n}, t>0 \\
x \in z+t E}} t^{\mu-n} \int_{z+t S}|f(y)| d y \\
& \leq \sum_{j}\left|R_{j}\right|^{1-\frac{\mu}{n}}\left(\sup _{\substack{z \in \mathbb{R}^{n}, t>0 \\
x \in z+t E}}\left|t R_{j}\right|^{\frac{\mu}{n}-1} \int_{z+t R_{j}}|f(y)| d y\right)
\end{aligned}
$$

pois $\left|t R_{j}\right|=t^{n}\left|R_{j}\right|$ e $S \subset \cup_{j} R_{j}$. Como $E \subset R_{j}^{*}$, temos $z+t E \subset z+t R_{j}^{*}=$ $\left(z+t R_{j}\right)^{*}$ pela definição. Assim, na notação do teorema (3.55)

$$
M_{S, E, \mu} f(x) \leq \sum_{j}\left|R_{j}\right|^{1-\frac{\mu}{n}} M_{\frac{\mu}{n}, j} f(x),
$$

onde $M_{\frac{\mu}{n}, j}$ é a função maximal (3.52) usando os retângulos em $\mathcal{B}\left(R_{j}\right)$. Portanto,

$$
\begin{aligned}
\left\|M_{S, E, \mu} f\right\|_{L_{w}^{q}} & \leq \sum_{j}\left|R_{j}\right|^{1-\frac{\mu}{n}}\left\|M_{\frac{\mu}{n}, j} f\right\|_{L_{w}^{q}} \\
& \leq c \sum_{j}\left|R_{j}\right|^{1-\frac{\mu}{n}} C_{R_{j}}\left|R_{j}\right|^{\frac{\mu}{n}-1}\|f\|_{L_{v}^{p}} \\
& =c\left(\sum C_{R_{j}}\right)\|f\|_{L_{v}^{p}}
\end{aligned}
$$

por (3.67) e teorema (3.55).

Isto completa a prova do teorema (3.66).

\subsection{Desigualdade do tipo fraco para o opera- dor maximal não centrado $M_{S, E, \mu}$, caso $S$ estrelado em relação à origem.}

O seguinte teorema é em parte análogo ao teorema 3.10 . 
Teorema 3.68 Sejam $0 \leq \mu<n, 1<p \leq q<\infty, \alpha=\frac{\mu}{n}$, S um conjunto estrelado em relação a origem e $E$ um conjunto limitado. Com a notação do teorema 3.66 , suponhamos que $v$ e $w$ satisfaçam

$$
|R|^{\alpha-1} w\left(R^{*}\right)^{1 / q}\left(\int_{R} v^{-p^{\prime} / p} d y\right)^{1 / p^{\prime}} \leq C_{j}
$$

para todo $R \in \mathcal{B}\left(R_{j}\right)$ e que $\sum C_{j}=b<\infty$. Então $M_{S, E, \mu}$ satisfaz a estimativa do tipo fraco

$$
w\left(\left\{x: M_{S, E, \mu} f(x)>\lambda\right\}\right) \leq\left(\frac{c\|f\|_{L_{v}^{p}}}{\lambda}\right)^{q}
$$

onde c é um múltiplo da constante $b$.

Prova: A prova segue em grande parte como a prova do teorema 0.10 e, portanto, omitiremos alguns trechos.

Considerando a notação do lema 3.53 , temos:

Lema 3.71 Se wé um peso, então

$$
w\left(\left\{x ; M_{\alpha} f(x)>\lambda\right\}\right) \leq w\left(\left\{x ; M_{\alpha}^{d y, z} f(x)>\lambda / c\right\}\right)
$$

com $c$ dependendo de a e n, mas não de $\mathcal{B}$ ou $f$.

Prova: Fixemos $R \in \mathcal{B}$ e consideremos a coleção daqueles $R_{1} \in \mathcal{B}^{d y}$ cujos comprimentos dos lados são duas vezes o comprimento dos lados de $R$, respectivamente, e consideremos em $\mathbb{R}^{n}$ como a união de tais $R_{1}$. É claro que $\left|R_{1}\right| \approx|R|$ com constante de comparabilidade dependendo somente de $n$.

Seja

$$
E\left(R_{1}\right)=\left\{z \in \mathbb{R}^{n} ; R \subset R_{1}+z, R^{*} \subset\left(R_{1}+z\right)^{*}\right\} .
$$

Assim, se $x \in R^{*}$ e $z \in E\left(R_{1}\right)$, então

$$
|R|^{\alpha-1} \int_{R}|f(y)| d y \leq c\left|R_{1}+z\right|^{\alpha-1} \int_{R_{1}+z}|f(y)| d y \leq c M_{\alpha}^{d y, z} f(x),
$$

pois $\left|R_{1}+z\right|=\left|R_{1}\right| \approx|R|, R \subset R_{1}+z$ e $x \in\left(R_{1}+z\right)^{*}$. Tomando na expressão acima o supremo sobre $R \in \mathcal{B}$ com $x \in R^{*}$, temos

$$
M_{\alpha} f(x) \leq c M_{\alpha}^{d y, z} f(x) .
$$


Portanto,

$$
w\left(\left\{x ; M_{\alpha} f(x)>\lambda\right\}\right) \leq w\left(\left\{x ; M_{\alpha}^{d y, z} f(x)>\lambda / c\right\}\right)
$$

O seguinte teorema é a versão tipo fraco do teorema 3.55 .

Teorema 3.72 Sejam $1<p \leq q<\infty$ e $0 \leq \alpha<1$ e seja $M_{\alpha}$ o operador definido em (3.52). Então $M_{\alpha}$ satisfaz a estimativa do tipo fraco

$$
w\left(\left\{x \in \mathbb{R}^{n} ; M_{\alpha} f(x)>\lambda\right\}\right) \leq\left(\frac{c\|f\|_{L_{v}^{p}}}{\lambda}\right)^{q}
$$

se e somente se.

$$
|R|^{\alpha-1} w\left(R^{*}\right)^{1 / q}\left(\int_{R} v^{-p^{\prime} / p} d x\right)^{1 / p^{\prime}} \leq C, \quad \text { para } R \in \mathcal{B}
$$

com uma constante $C$ que é um múltiplo de $c$, dependendo de $\alpha, n, p, e q$, mas não de $\mathcal{B}$ ou $f$.

Prova: Observemos que para provar que (3.74) é condição suficiente para (3.73), é suficiente pelo lema 3.71 provar o análogo de (3.73) para $M_{\alpha}^{d y, z}$, com uma constante independente de $z$. Para isto, trocando $f$ por $f v^{-p^{\prime} / p}$ em (3.73) temos

$$
w\left(\left\{x \in \mathbb{R}^{n} ; M_{\alpha}^{d y, z} f v^{-p^{\prime} / p}(x)>\lambda\right\}\right) \leq\left(\frac{c\|f\|_{L_{v^{-} p^{\prime} / p}}}{\lambda}\right)^{q},
$$

com $c$ um múltiplo da constante em (3.73) dependendo somente de $\alpha, n, p$, e $q$. Para provar (3.75) tomemos os conjuntos $\Omega_{k}$ como na prova do teorema 3.55 , lembrando que valem então (3.60) e (3.61) e que por (3.61),

$$
\Omega_{k} \backslash \Omega_{k+1}=\cup_{j}\left[\left(R_{j}^{k}+z\right)^{*} \backslash \Omega_{k+1}\right]=\cup_{j} E_{j}^{k},
$$

onde

$$
E_{j}^{k}=\left(R_{j}^{k}+z\right)^{*} \backslash \Omega_{k+1}
$$

Para $\lambda>0$, seja

$$
\Omega_{\lambda}=\left\{x \in \mathbb{R}^{n} ; M_{\alpha}^{d y, z}\left(f v^{-p^{\prime} / p}\right)(x)>\lambda\right\} .
$$


Tomemos $k_{0} \in \mathbb{Z}$ tal que

$$
2^{\left(k_{0}+1\right) n} \geq \lambda \geq 2^{k_{0} n} .
$$

$\operatorname{Logo} \lambda^{-1} \approx 2^{-k_{0} n}$ e $\Omega_{\lambda} \subset \Omega_{k_{0}}$.

Utilizando todos os resultados obtidos acima, temos:

$$
\begin{aligned}
& w\left(\Omega_{\lambda}\right) \leq w\left(\Omega_{k_{0}}\right)=w\left(\cup_{k=k_{0}}^{\infty} \Omega_{k} \backslash \Omega_{k+1}\right)=w\left(\cup_{k=k_{0}}^{\infty} \cup_{j=1}^{\infty} E_{j}^{k}\right) \\
& \leq \sum_{\substack{k=k_{0} \\
j=1}}^{\infty} w\left(E_{j}^{k}\right) \leq \sum_{\substack{k=k_{0} \\
j=1}}^{\infty}\left(2^{-k n}\left|R_{j}^{k}\right|^{\alpha-1} \int_{R_{j}^{k}+z} f v^{-p^{\prime} / p} d x\right)^{q} w\left(E_{j}^{k}\right) \\
& \leq \sum_{\substack{k=k_{0} \\
j=1}}^{\infty} w\left(\left(R_{j}^{k}+z\right)^{*}\right)\left(2^{-k n}\left|R_{j}^{k}\right|^{\alpha-1} \int_{R_{j}^{k}+z} f v^{-p^{\prime} / p} d x\right)^{q} \\
& \leq \lambda^{-q} \sum_{\substack{k=k_{0} \\
j=1}}^{\infty} w\left(\left(R_{j}^{k}+z\right)^{*}\right)\left(\left|R_{j}^{k}\right|^{\alpha-1} 2^{-\left(k-k_{0}\right) n}\right)^{q}\left(\int_{R_{j}^{k}+z} f v^{-p^{\prime} / p} d x\right)^{q} \\
& =\lambda^{-q} \sum_{\substack{k=k_{0} \\
j=1}}^{\infty} w\left(\left(R_{j}^{k}+z\right)^{*}\right)\left(\left|R_{j}^{k}\right|^{\alpha-1} 2^{-\left(k-k_{0}\right) n}\right)^{q} . \\
& \cdot\left(\int_{R_{j}^{k}+z} f\left(v^{-p^{\prime} / p}\right)^{1 / p}\left(v^{-p^{\prime} / p}\right)^{1 / p^{\prime}} d x\right)^{q} \\
& \leq \lambda^{-q} \sum_{\substack{k=k_{0} \\
j=1}}^{\infty} w\left(\left(R_{j}^{k}+z\right)^{*}\right)\left(\left|R_{j}^{k}\right|^{\alpha-1} 2^{-\left(k-k_{0}\right) n}\left(\int_{R_{j}^{k}+z} v^{-p^{\prime} / p}\right)^{1 / p^{\prime}}\right)^{q} . \\
& \left(\int_{R_{j}^{k}+z} f^{p} v^{-p^{\prime} / p} d x\right)^{q / p} \\
& \leq \lambda^{-q} C^{q} \sum_{\substack{k=k_{0} \\
j=1}}^{\infty} 2^{-\left(k-k_{0}\right) n q}\left(\int_{R_{j}^{k}+z} f^{p} v^{-p^{\prime} / p} d x\right)^{q / p} \quad \text { por }(3.74)
\end{aligned}
$$




$$
\begin{aligned}
& =\lambda^{-q} C^{q} \sum_{k=K_{0}}^{\infty} 2^{-\left(k-k_{0}\right) n q}\left(\sum_{j=1}^{\infty} \int_{R_{j}^{k}+z} f^{p} v^{-p^{\prime} / p} d x\right)^{q / p} \\
& \leq \lambda^{-q} C^{q} \sum_{m=0}^{\infty}\left(2^{-n q}\right)^{m}\|f\|_{L_{v}^{p}-p^{\prime} / p}^{q} \text { onde } m=k-k_{0} \\
& =\lambda^{-q} C^{q}\left(\frac{2^{n q}}{2^{n q}-1}\right)\|f\|_{L_{v}^{p}-p^{\prime} / p}^{q}
\end{aligned}
$$

o que conclui a prova de (3.75).

Para provar a parte recíproca basta tomar $f=\chi_{R} v^{-p^{\prime} / p}$ e usar o mesmo argumento "standard" usado na prova do teorema de Muckenhoupt.

Finalmente, provemos o teorema 3.68.

Assim como na prova do teorema 3.66 , temos

$$
M_{S, E, \mu} f(x) \leq \sum_{j}\left|R_{j}\right|^{1-\frac{\mu}{n}} M_{\frac{\mu}{n}, j} f(x) .
$$

Logo;

$$
\begin{aligned}
w\left(\left\{x ; M_{S, E, \mu} f(x)>\lambda\right\}\right) & =\int_{\left\{x ; M_{S, E, \mu} f(x)>\lambda\right\}} w d x \\
& \leq \int_{\left\{x ; \sum_{j}\left|R_{j}\right|^{1-\frac{\mu}{n}} M_{\frac{\mu}{n}, j} f(x)>\lambda\right\}} w d x \\
& \leq \int_{\cup_{j}\left\{x ;\left|R_{j}\right|^{1-\frac{\mu}{n}} M_{\frac{\mu}{n}, j} f(x)>\lambda / 2^{j}\right\}} w d x \\
& \leq \sum_{j=1}^{\infty} \int_{\left\{x ; M_{\frac{\mu}{n}, j} f(x)>\frac{\lambda}{2^{j}\left|R_{j}\right|^{1-\frac{\mu}{n}}}\right\}} w d x \\
& =\sum_{j=1}^{\infty} w\left(\left\{x ; M_{\frac{\mu}{n}, j} f(x)>\frac{\lambda}{2^{j}\left|R_{j}\right|^{1-\frac{\mu}{n}}}\right\}\right) \\
& \leq \sum_{j=1}^{\infty}\left[C_{j}\left|R_{j}\right|^{1-\frac{\mu}{n}}\right]^{q} \frac{\|f\|_{L_{v}^{p}}^{q}}{\lambda^{q}} 2^{-q j}\left|R_{j}\right|^{\left(\frac{\mu}{n}-1\right) q} \\
& \leq\left(\sum_{j=1}^{\infty} 2^{-j} C_{j}\right)^{q}\left[\frac{\|f\|_{L_{v}^{p}}}{\lambda}\right]^{q}=C^{\prime}\left[\frac{\|f\|_{L_{v}^{p}}}{\lambda}\right]^{q} .
\end{aligned}
$$


A segunda desigualdade acima segue pelo seguinte fato:

Se $\sum_{j=1}^{\infty} g_{j}(x)>\lambda$ então existe $j$ tal que $g_{j}(x)>\lambda / 2^{j}$. De fato, pois se para qualquer $j, g_{j}(x) \leq \lambda / 2^{j}$, então

$$
\sum_{j=1}^{\infty} g_{j}(x) \leq \sum_{j=1}^{\infty} \lambda / 2^{j}=\lambda
$$

o que é uma contradição. Logo,

$$
\left\{x ; \sum_{j=1}^{\infty} g_{j}(x)>\lambda\right\} \subset \bigcup_{j}\left\{x ; g_{j}(x)>\lambda / 2^{j}\right\},
$$

concluindo assim a prova do teorema 3.68 . 


\section{Referências Bibliográficas}

[Ca] C. P. Calderón, Differentiation through starlike sets in $\mathbb{R}^{m}$, Studia Math. 48 (1973), 1-13.

[CWW] S. Chanillo, D. K. Watson e R. L. Wheeden, Some integral and maximal operators related to starlike sets, Studia Math. 107 (1993), 223-255.

[FZ] J. C. D. Fernandes e S. L. Zani, Desigualdades de Poincaré e Sobolev com pesos, $20^{\circ}$ Col. Bras. Mat.-IMPA, (1995).

[H] R. A. Hunt, On $L(p, q)$ Spaces, Enseignement Math. (2) 12 (1966), 249-276.

[J] B. Jawerth, Weighted inequalities for maximal operators: linearization, localization, and factorization, Amer. J. Math. 108 (1986), 361-414.

[M] B. Muckenhoupt, Weighted norm inequalities for the Hardy Maximal Function, Trans. Amer. Math. Soc. 165 (1972), 207-226.

[MW] B. Muckenhoupt e R. L. Wheeden, Weighted norm inequalities for fractional integrals, Trans. Amer. Math. Soc. 192 (1974), 261-274.

[P1] C. Pérez, Two weighted inequalities for potential and fractional type maximal operators, Indiana Univ. Math. J. 43 (1994), 663-683.

[P2] ——, A remark on weighted inequalities for general maximal operators, Proc. Amer. Math. Soc. 119 (1993), 1121-1126.

[SW] E. Sawyer e R. L. Wheeden, Weighted inequalities for fractional integrals on Euclidean and Homogeneous Spaces, Amer. J. Math. 114 (1992), 813-874. 
[StWe] E. M. Stein e N. J. Weiss, On the convergence of Poisson integrals, Trans. Amer. Math. Soc. 140 (1969), 35-54.

[T] A. Torchinsky, Real-Variable Methods in Harmonic Analysis, Academic Press Inc., New York, (1986).

[W] R. L. Wheeden, Norm inequalities for off centered maximal operators, Publ. Mat. 37 (1993), no 2, 429-441.

[WZ] R. L. Wheeden e A. Zygmund, Measure and Integral - An introduction to Real Analysis, Marcel Dekker Inc., New York (1977). 